\title{
Structural identifiability analysis of a cardiovascular system model
}

\author{
A. Pironet, P.C. Dauby, J.G. Chase, \\ J.A. Revie, P.D. Docherty, T. Desaive
}




\section{Introduction}

- To be clinically relevant, mathematical models have to be made patient-specific. 


\section{Introduction}

- To be clinically relevant, mathematical models have to be made patient-specific.

- Can we find a measurement set which allows to identify all model parameters? 


\section{Introduction}

- To be clinically relevant, mathematical models have to be made patient-specific.

- Can we find a measurement set which allows to identify all model parameters?

- Structural identifiability analysis. 


\section{Introduction}

- To be clinically relevant, mathematical models have to be made patient-specific.

- Can we find a measurement set which allows to identify all model parameters?

- Structural identifiability analysis.

- Goal: investigate structural identifiability of the six-chamber CVS model from a clinically available measurement set. 


\section{Six-chamber CV system model}

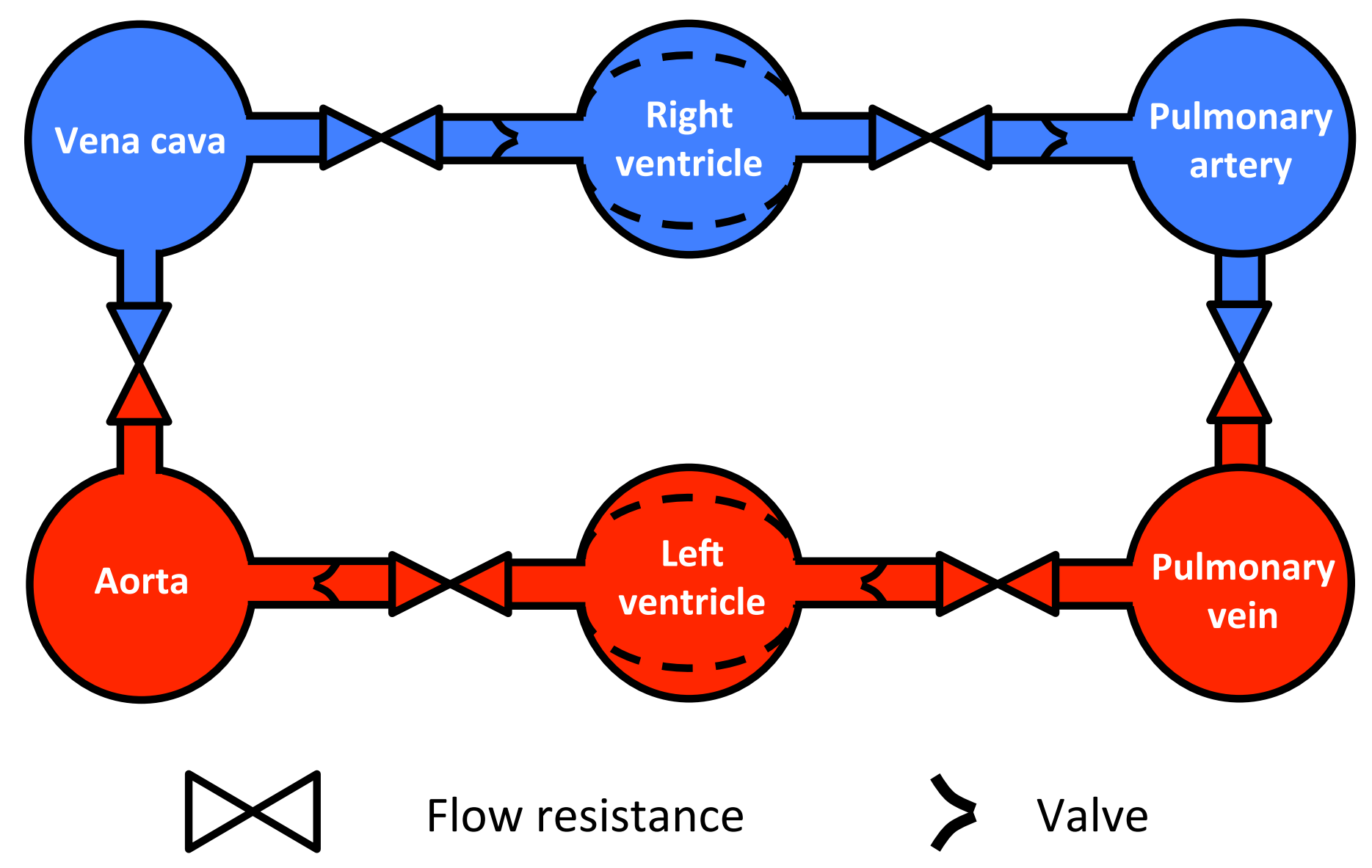




\section{Six-chamber CV system model}

Passive chambers:
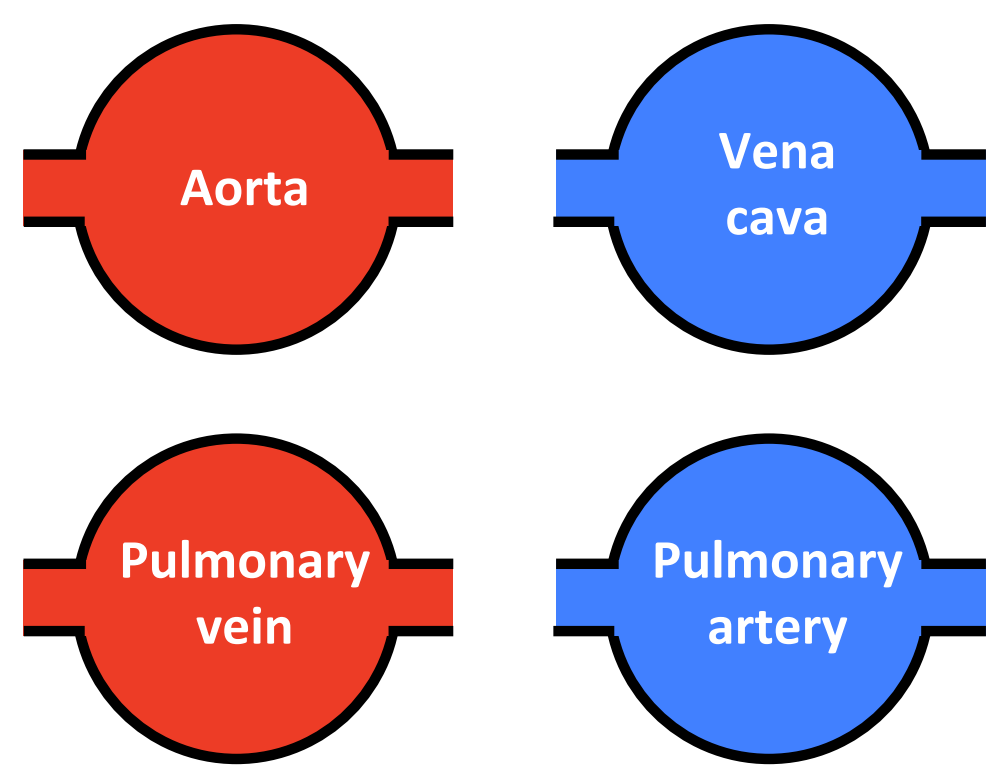

$$
\mathrm{P}=\mathrm{E} \cdot \mathrm{V}
$$

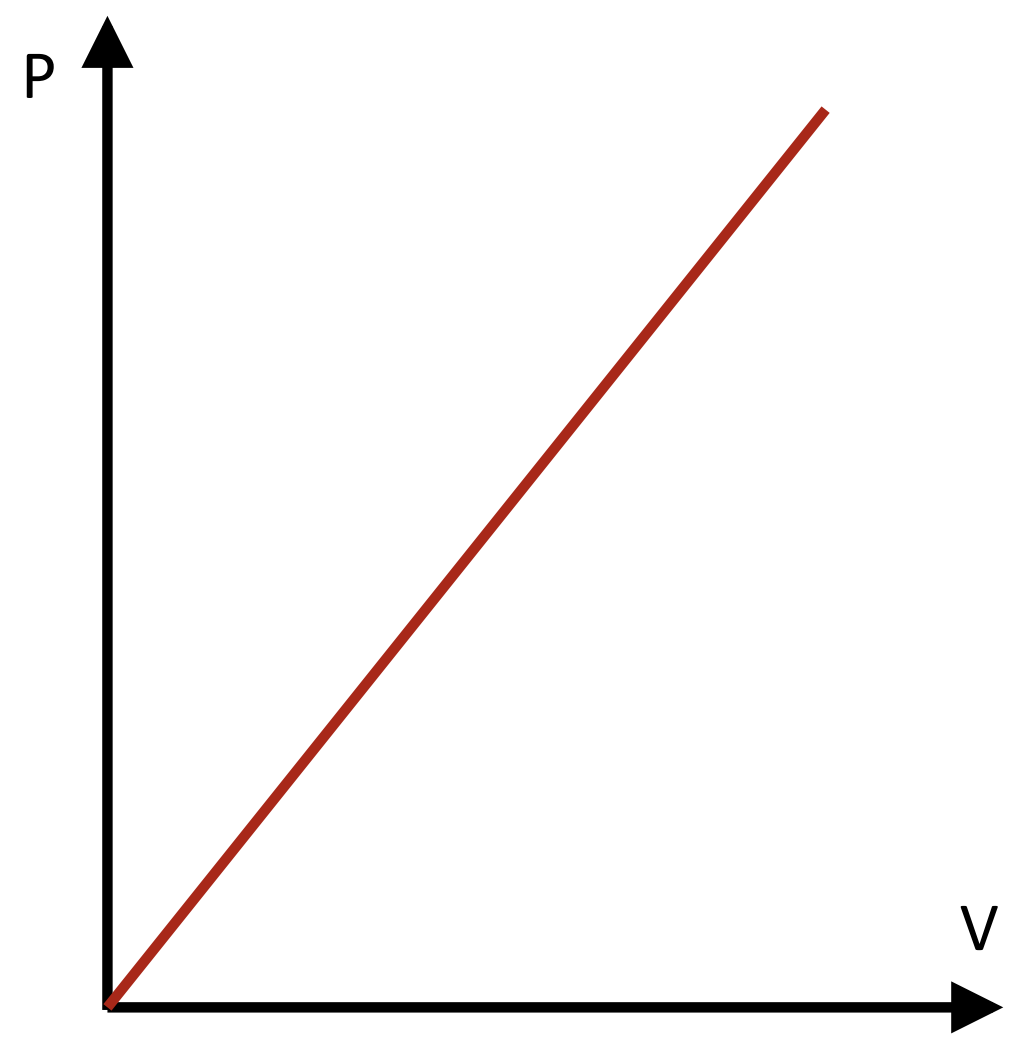




\section{Six-chamber CV system model}

Passive chambers:
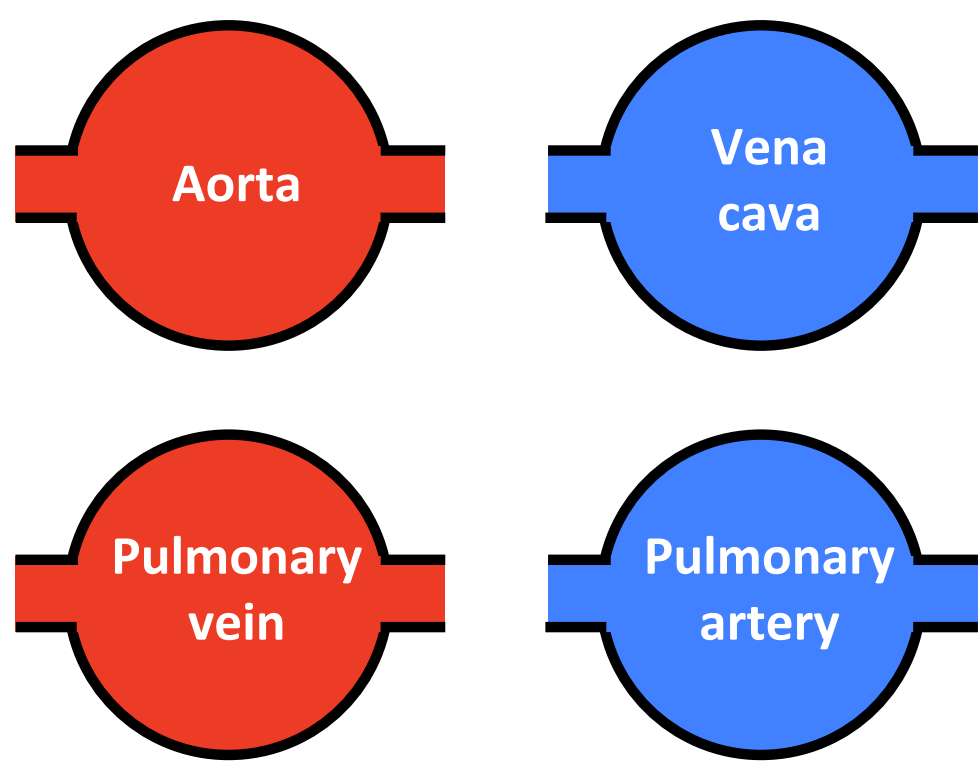

$$
\begin{aligned}
& P_{\mathrm{ao}}=\mathrm{E}_{\mathrm{ao}} \cdot \mathrm{V}_{\mathrm{ao}} \\
& \mathrm{P}_{\mathrm{vc}}=\mathrm{E}_{\mathrm{vc}} \cdot \mathrm{V}_{\mathrm{vc}} \\
& \mathrm{P}_{\mathrm{pu}}=\mathrm{E}_{\mathrm{pu}} \cdot \mathrm{V}_{\mathrm{pu}} \\
& \mathrm{P}_{\mathrm{pa}}=\mathrm{E}_{\mathrm{pa}} \cdot \mathrm{V}_{\mathrm{pa}}
\end{aligned}
$$

$$
\mathrm{P}=\mathrm{E} \cdot \mathrm{V}
$$




\section{Six-chamber CV system model}

Cardiac chambers:

$$
P=E \cdot e \cdot V
$$
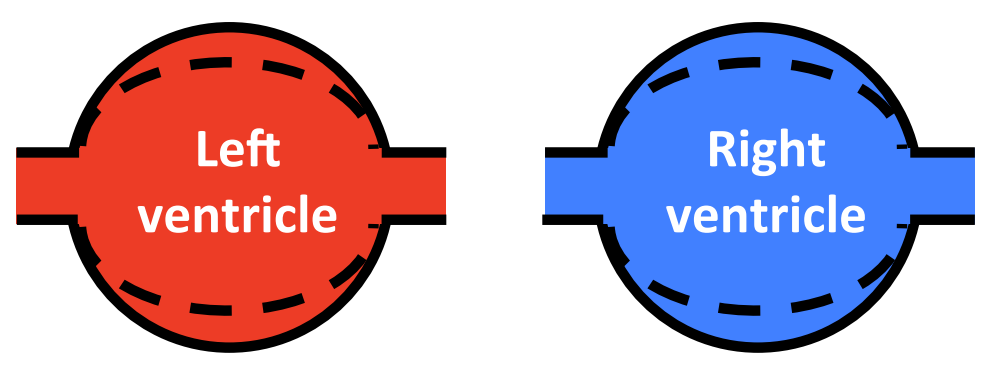


\section{Six-chamber CV system model}

Cardiac chambers:
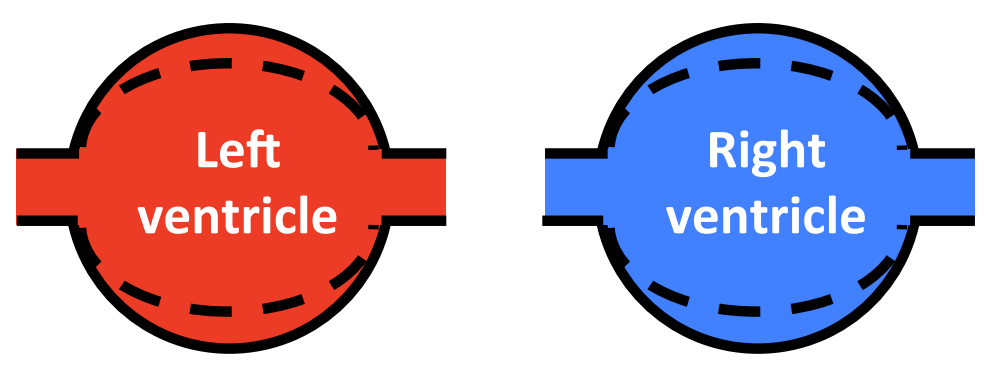

$$
P=E \cdot e \cdot V
$$

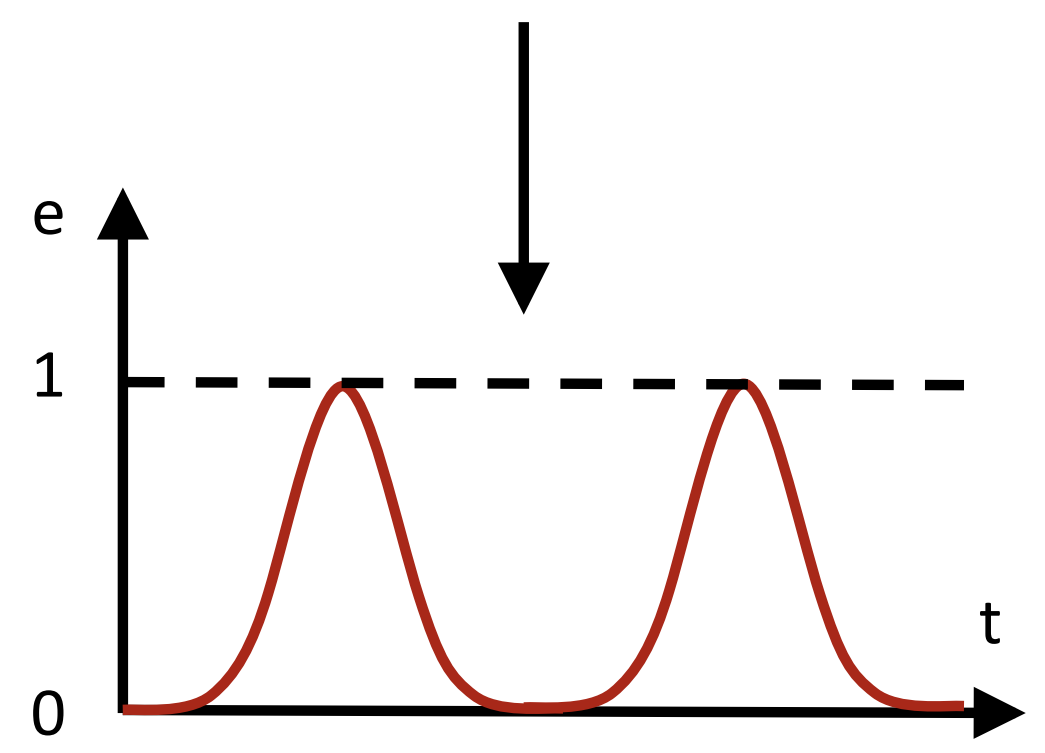

Driver function 


\section{Six-chamber CV system model}

Cardiac chambers:

$$
\mathrm{P}=\mathrm{E} \cdot \mathrm{e} \cdot \mathrm{V}
$$
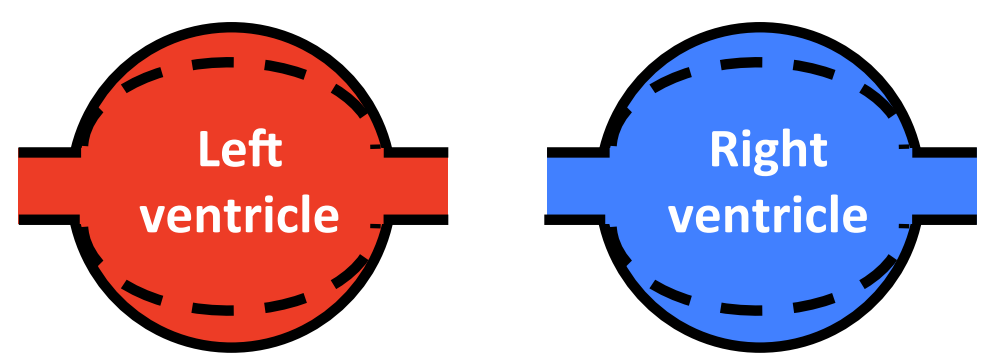

$$
\begin{aligned}
& P_{l v}=E_{l v} \cdot e_{l v} \cdot V_{l v} \\
& P_{r v}=E_{r v} \cdot e_{r v} \cdot V_{r v}
\end{aligned}
$$

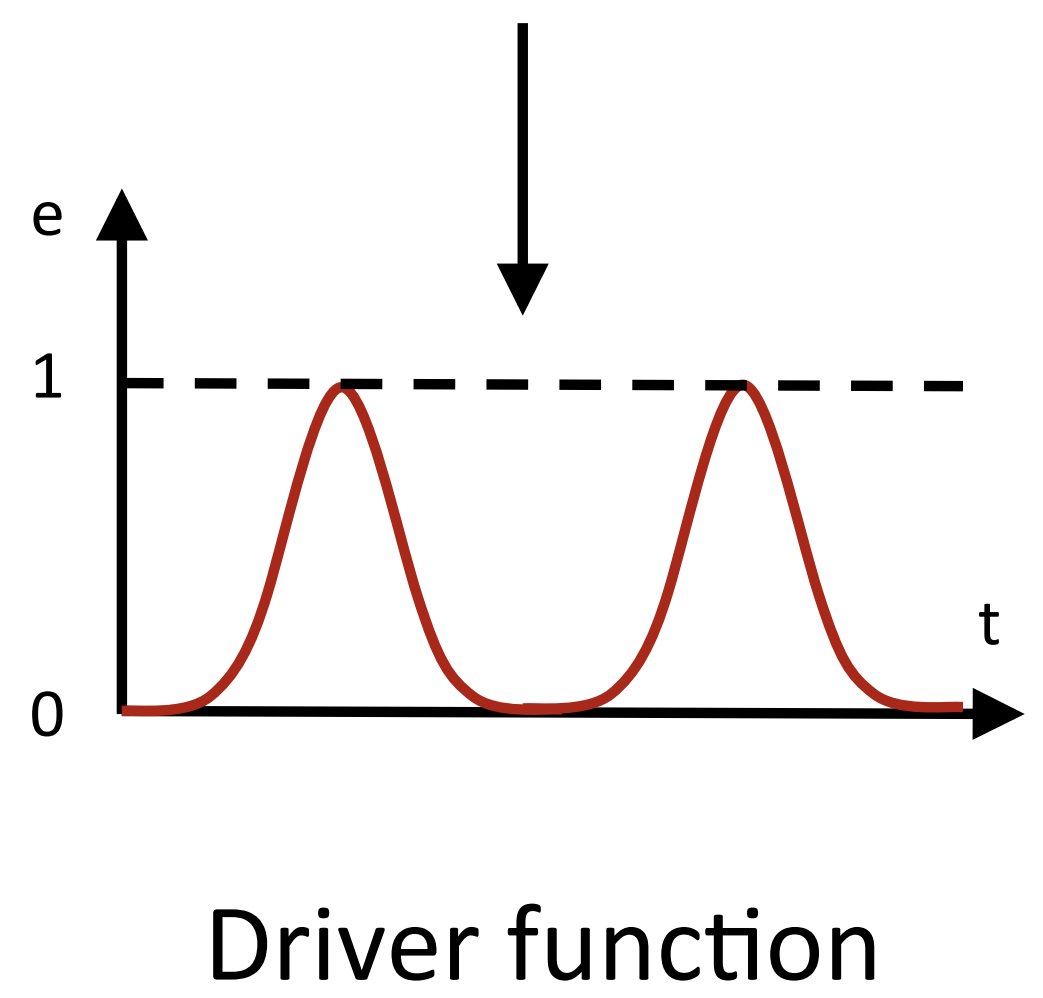




\section{Six-chamber CV system model}

No valve:
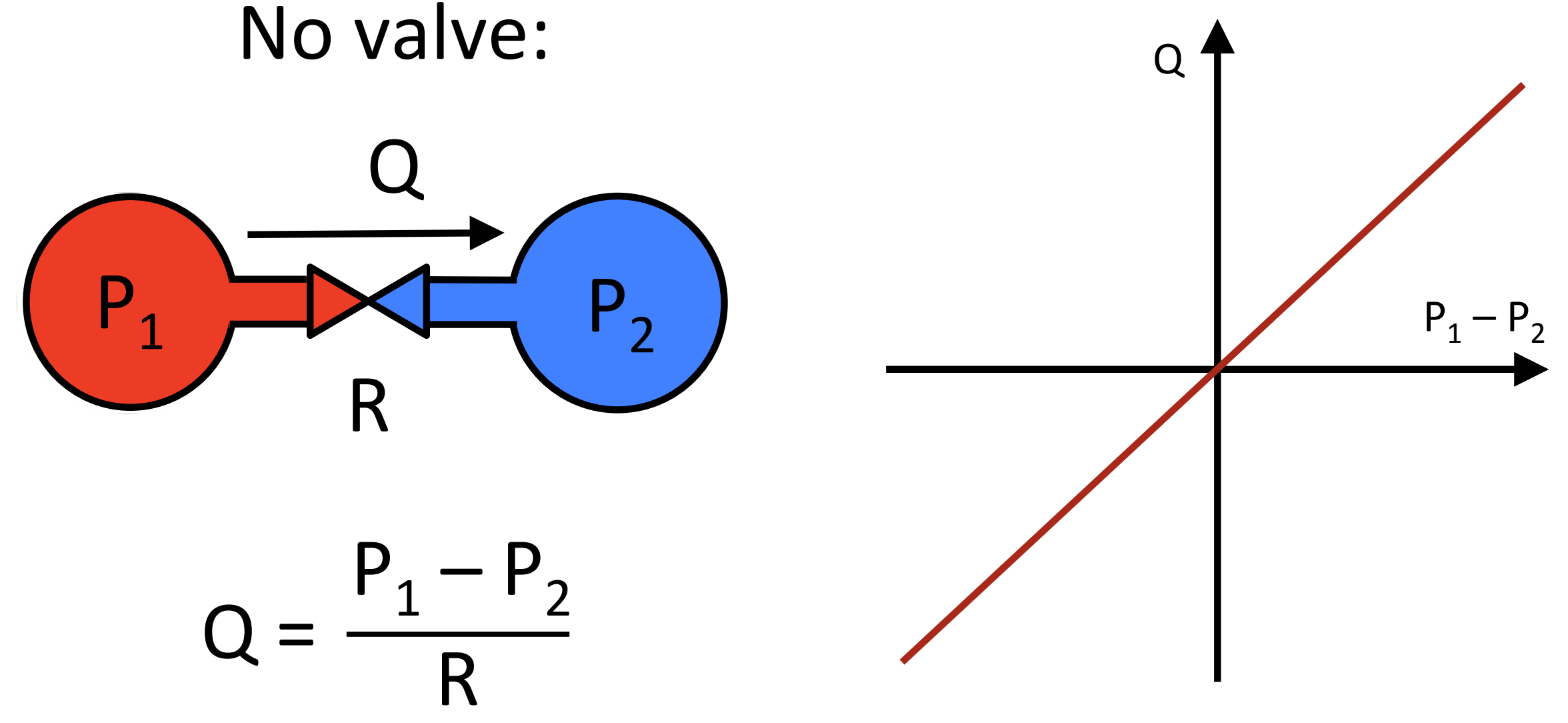


\section{Six-chamber CV system model}

No valve:

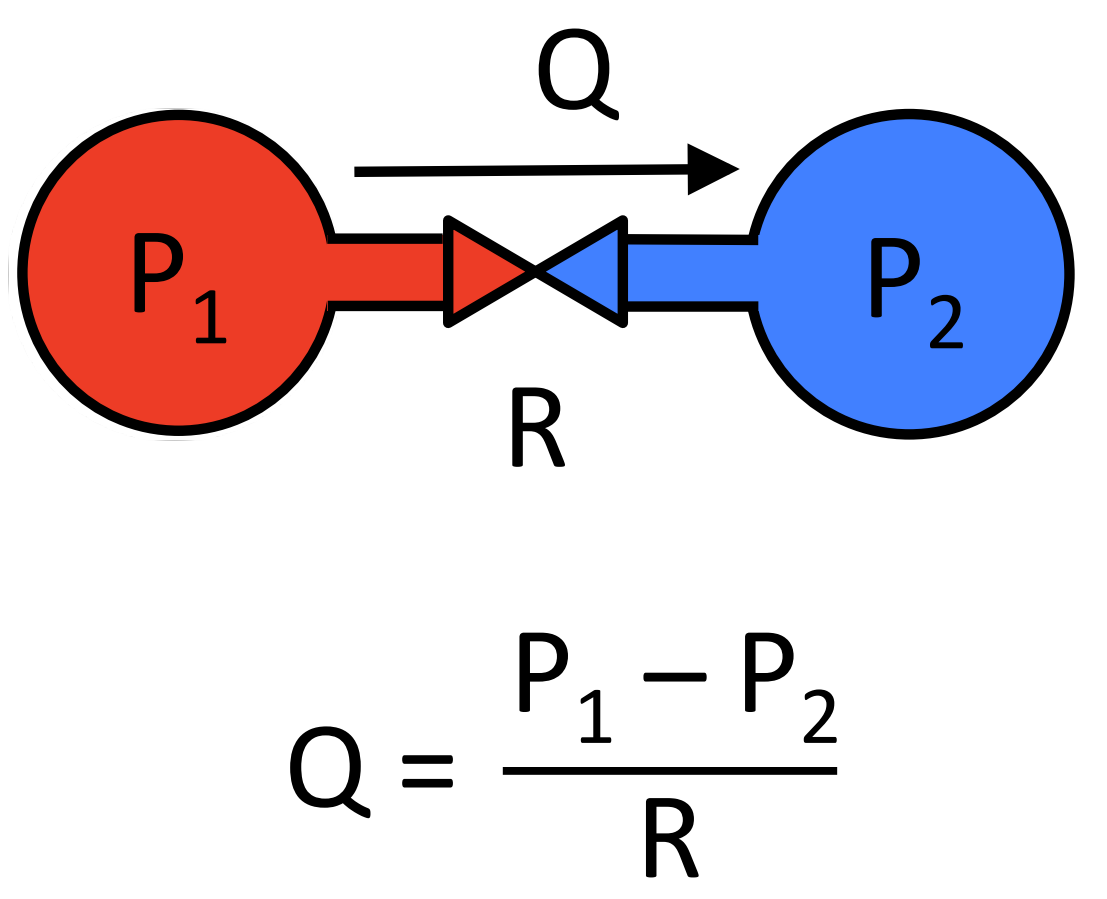

$$
\begin{aligned}
& Q_{\text {sys }}=\frac{P_{a o}-P_{v c}}{R_{s y s}} \\
& Q_{p u l}=\frac{P_{p a}-P_{p u}}{R_{p u l}}
\end{aligned}
$$




\section{Six-chamber CV system model}

Valve:

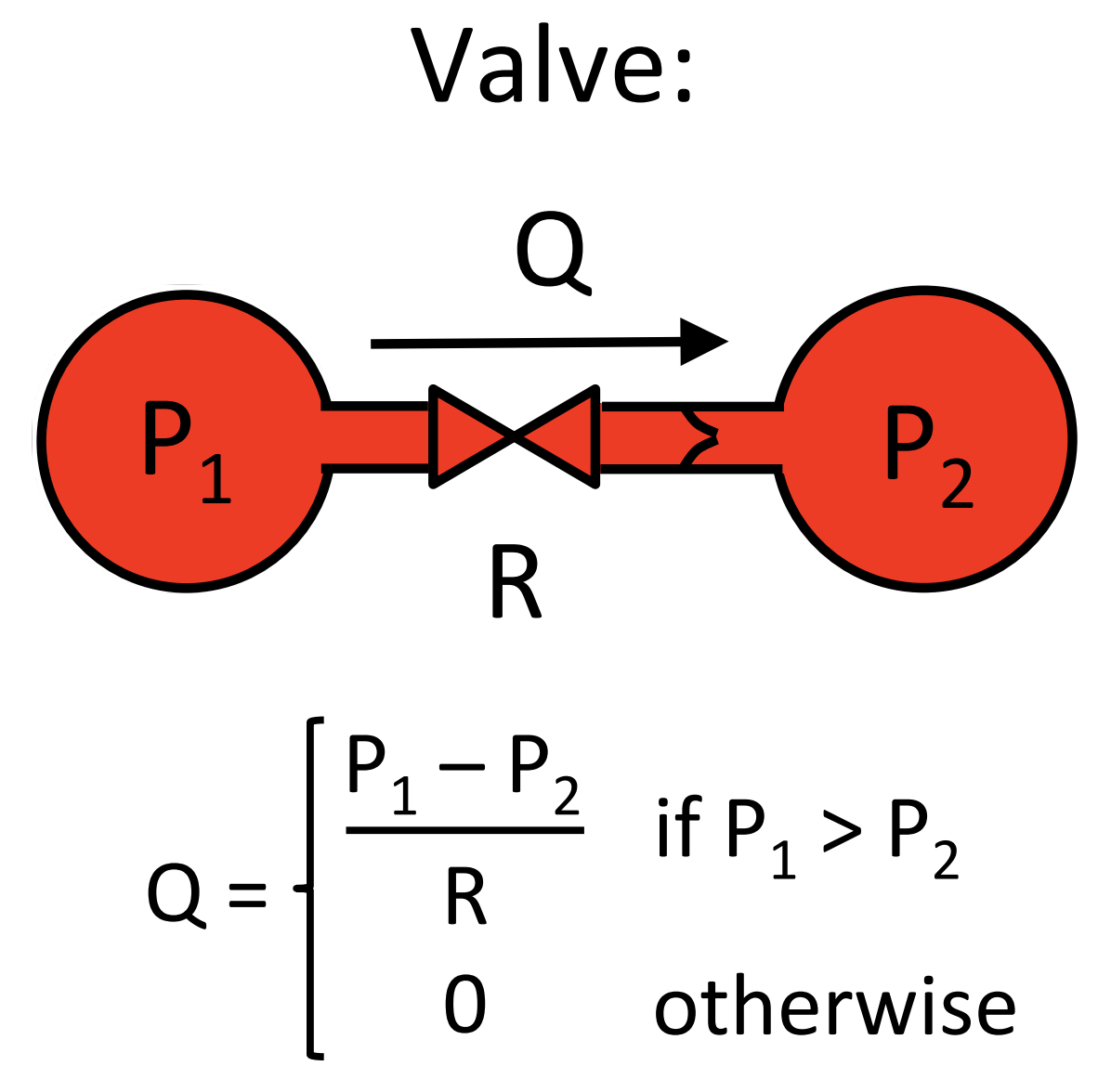

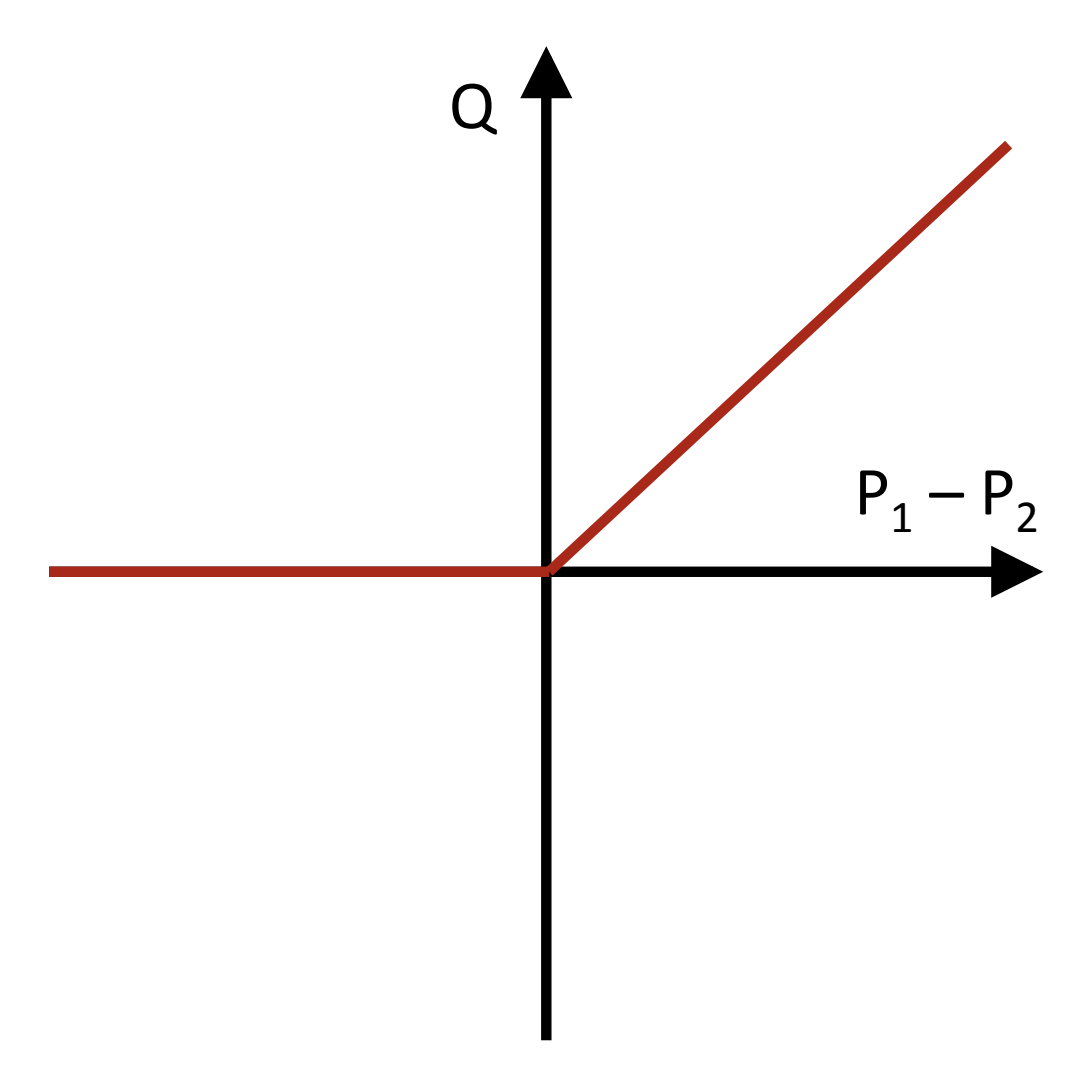




\section{Six-chamber CV system model}

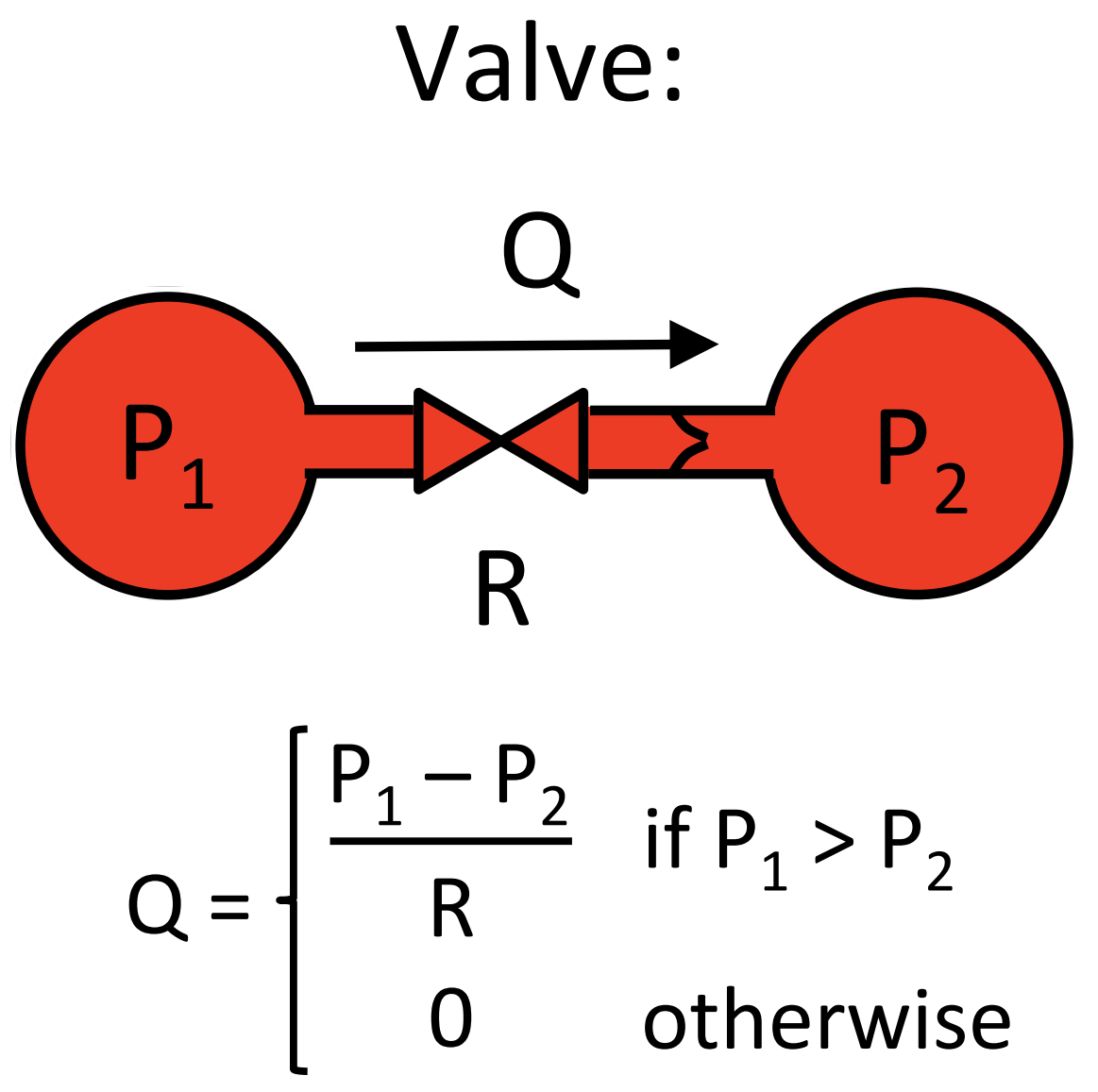

$$
\begin{aligned}
& Q_{m t}=\frac{r\left(P_{p u}-P_{l v}\right)}{R_{m t}} \\
& Q_{a v}=\frac{r\left(P_{l v}-P_{a o}\right)}{R_{a v}} \\
& Q_{t c}=\frac{r\left(P_{v c}-P_{r v}\right)}{R_{t c}} \\
& Q_{p v}=\frac{r\left(P_{r v}-P_{p a}\right)}{R_{p v}}
\end{aligned}
$$


Six-chamber CV system model Continuity equation:

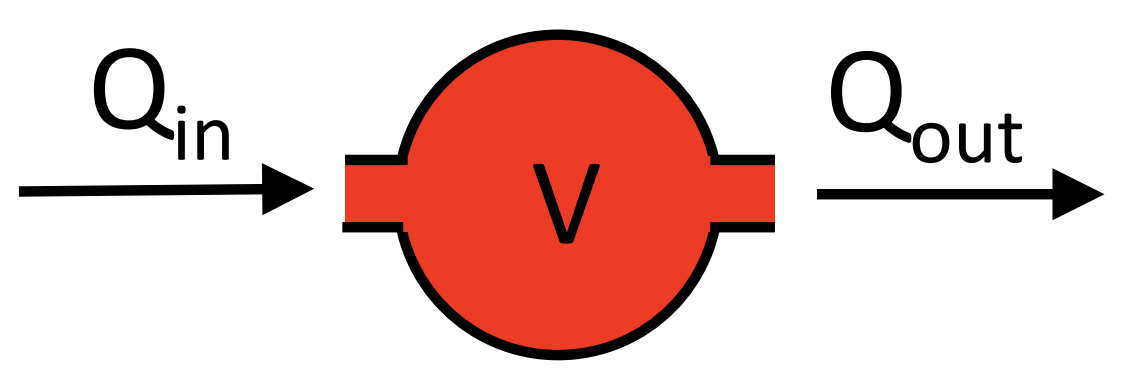

$$
\dot{V}=Q_{\text {in }}-Q_{\text {out }}
$$




\section{Six-chamber CV system model}

\section{Continuity equation:}

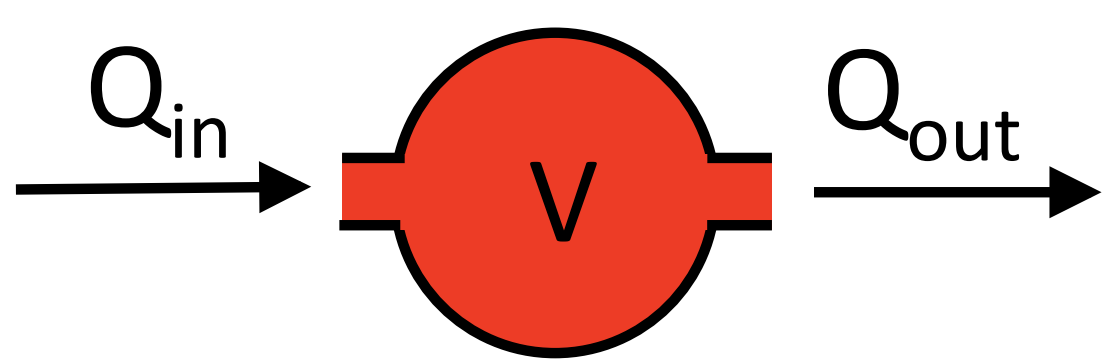

$$
\begin{aligned}
& \dot{\mathrm{V}}_{\mathrm{lv}}=\mathrm{Q}_{\mathrm{mt}}-\mathrm{Q}_{\mathrm{av}} \\
& \dot{\mathrm{V}}_{\mathrm{ao}}=\mathrm{Q}_{\mathrm{av}}-\mathrm{Q}_{\mathrm{sys}} \\
& \dot{\mathrm{V}}_{\mathrm{vc}}=\mathrm{Q}_{\mathrm{sys}}-\mathrm{Q}_{\mathrm{tc}} \\
& \dot{\mathrm{V}}_{\mathrm{rv}}=\mathrm{Q}_{\mathrm{tc}}-\mathrm{Q}_{\mathrm{pv}} \\
& \dot{\mathrm{V}}_{\mathrm{pa}}=\mathrm{Q}_{\mathrm{pv}}-\mathrm{Q}_{\mathrm{pul}} \\
& \dot{\mathrm{V}}_{\mathrm{pu}}=\mathrm{Q}_{\mathrm{pul}}-\mathrm{Q}_{\mathrm{mt}}
\end{aligned}
$$$$
\dot{\mathrm{V}}=\mathrm{Q}_{\text {in }}-\mathrm{Q}_{\text {out }}
$$ 


\section{Six-chamber CV system model}

\section{Continuity equation:}

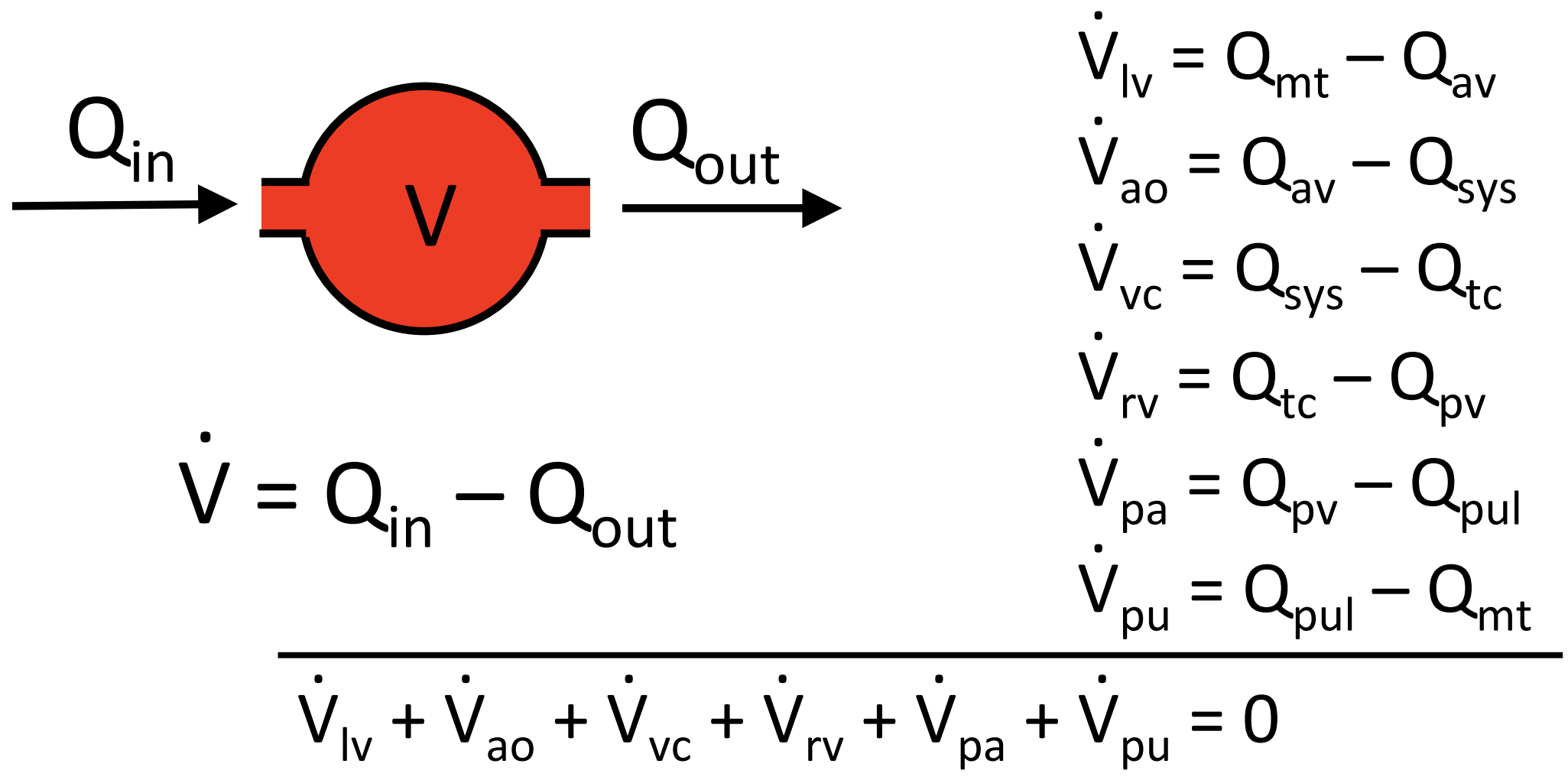




\section{Six-chamber CV system model}

\section{Continuity equation:}

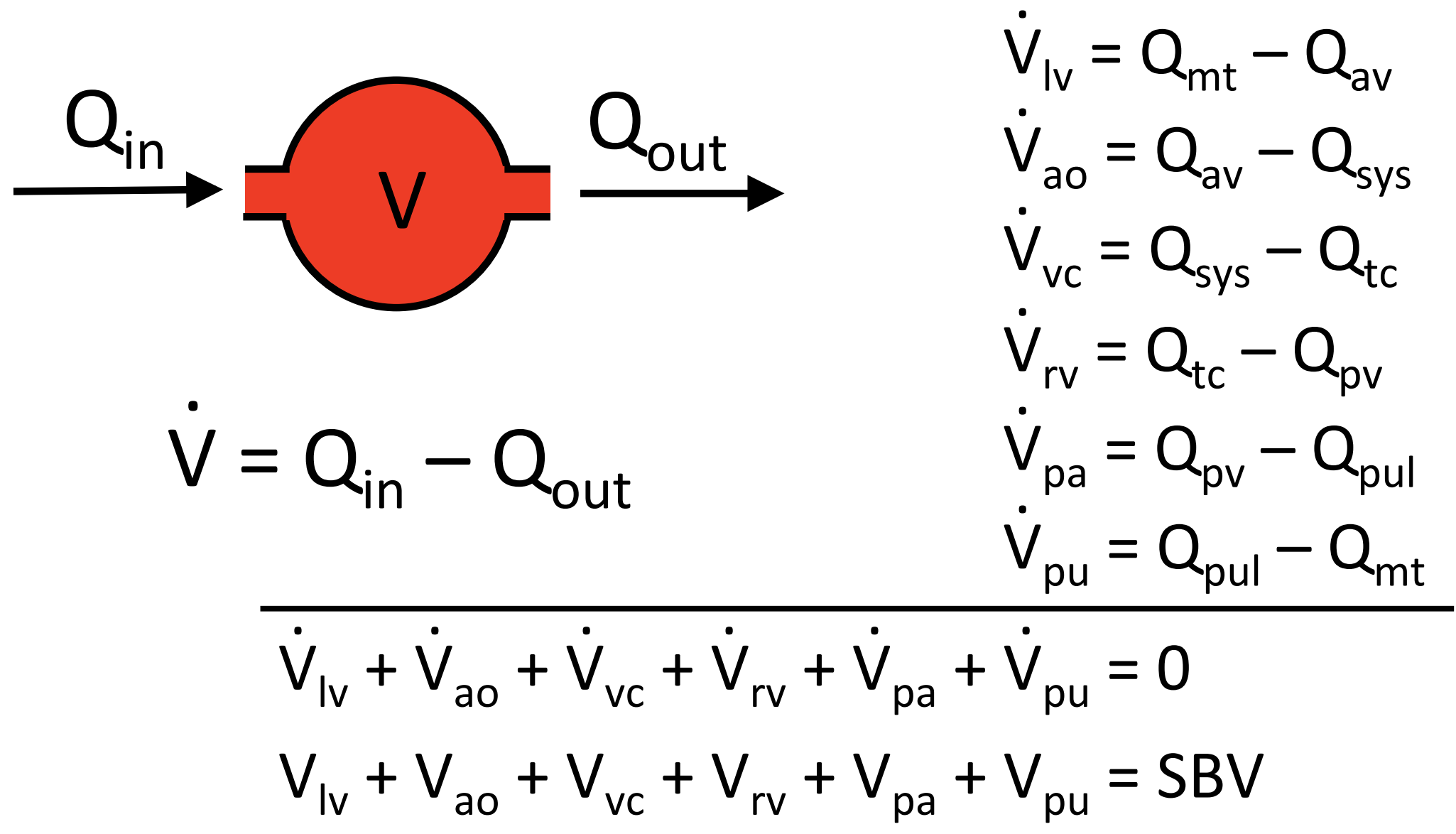




\section{Identifiability of the CV system model}

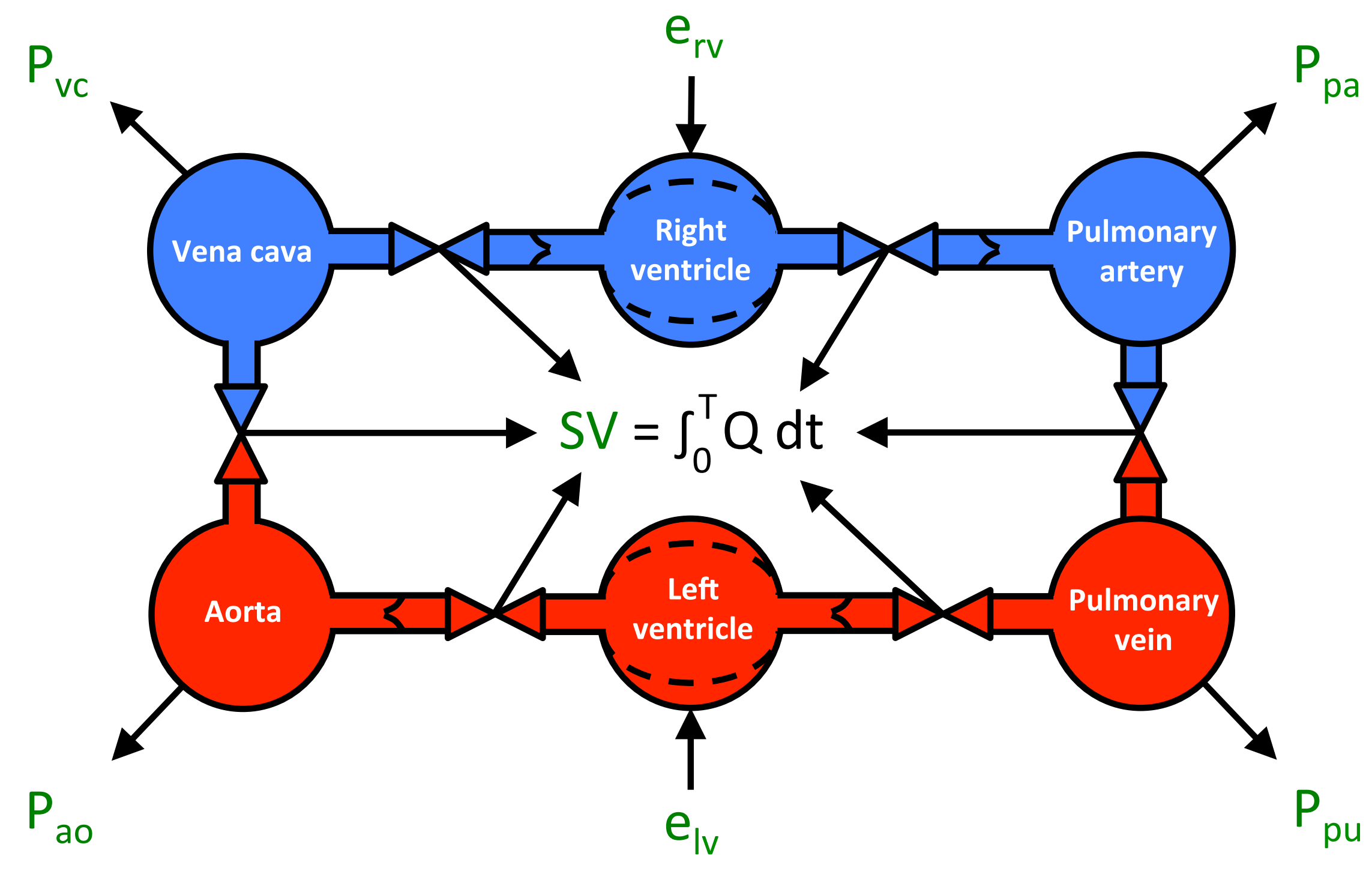




\section{During the whole cardiac cycle}

$\dot{\mathrm{V}}_{\mathrm{lv}}=\mathrm{Q}_{\mathrm{mt}}-\mathrm{Q}_{\mathrm{av}}$

$\dot{\mathrm{V}}_{\mathrm{ao}}=\mathrm{Q}_{\mathrm{av}}-\mathrm{Q}_{\mathrm{sys}}$

$\dot{\mathrm{V}}_{\mathrm{vc}}=\mathrm{Q}_{\mathrm{sys}}-\mathrm{Q}_{\mathrm{tc}}$

$\dot{\mathrm{V}}_{\mathrm{rv}}=\mathrm{Q}_{\mathrm{tc}}-\mathrm{Q}_{\mathrm{pv}}$

$\dot{\mathrm{V}}_{\mathrm{pa}}=\mathrm{Q}_{\mathrm{pv}}-\mathrm{Q}_{\mathrm{pul}}$

$\dot{\mathrm{V}}_{\mathrm{pu}}=\mathrm{Q}_{\mathrm{pul}}-\mathrm{Q}_{\mathrm{mt}}$

$\mathrm{SBV}=\mathrm{V}_{\mathrm{lv}}+\mathrm{V}_{\mathrm{ao}}+\mathrm{V}_{\mathrm{vc}}+\mathrm{V}_{\mathrm{rV}}+\mathrm{V}_{\mathrm{pa}}+\mathrm{V}_{\mathrm{pu}}$

$S V=\int_{0}^{T} Q d t$

$$
\begin{aligned}
& Q_{m t}=\frac{r\left(P_{p u}-P_{l v}\right)}{R_{m t}} \\
& Q_{a v}=\frac{r\left(P_{l v}-P_{a o}\right)}{R_{a v}} \\
& Q_{s y s}=\frac{P_{a o}-P_{v c}}{R_{s y s}} \\
& Q_{t c}=\frac{r\left(P_{v c}-P_{r v}\right)}{R_{t c}} \\
& Q_{p v}=\frac{r\left(P_{r v}-P_{p a}\right)}{R_{p v}} \\
& Q_{p u l}=\frac{P_{p a}-P_{p u}}{R_{p u l}}
\end{aligned}
$$




\section{During the whole cardiac cycle}

$$
Q_{s y s}=\frac{P_{a o}-P_{v c}}{R_{s y s}}
$$

$S V=\int_{0}^{T} Q d t$ 


\section{During the whole cardiac cycle}

$S V=\int_{0}^{T} Q d t$

$$
\begin{array}{r}
Q_{\text {sys }}=\frac{P_{\text {ao }}-P_{v c}}{R_{\text {sys }}} \\
\int_{0}^{T} Q_{\text {sys }} d t=\frac{\int_{0}^{T}\left(P_{a o}-P_{v c}\right) d t}{R_{\text {sys }}}
\end{array}
$$




\section{During the whole cardiac cycle}

$$
Q_{\text {sys }}=\frac{P_{\text {ao }}-P_{v c}}{R_{\text {sys }}}
$$

$S V=\int_{0}^{T} Q d t \longrightarrow S V=\frac{\int_{0}^{T}\left(P_{a o}-P_{v c}\right) d t}{R_{\text {sys }}}$ 


\section{During the whole cardiac cycle}

$S V=\int_{0}^{T} Q d t$

$$
\begin{array}{r}
Q_{\text {sys }}=\frac{P_{a o}-P_{v c}}{R_{s y s}} \\
R_{s y s}=\frac{\int_{0}^{T}\left(P_{a o}-P_{v c}\right) d t}{S V}
\end{array}
$$




\section{During the whole cardiac cycle}

$\dot{\mathrm{V}}_{\mathrm{lv}}=\mathrm{Q}_{\mathrm{mt}}-\mathrm{Q}_{\mathrm{av}}$

$\dot{\mathrm{V}}_{\mathrm{ao}}=\mathrm{Q}_{\mathrm{av}}-\mathrm{Q}_{\mathrm{sys}}$

$\dot{\mathrm{V}}_{\mathrm{vc}}=\mathrm{Q}_{\mathrm{sys}}-\mathrm{Q}_{\mathrm{tc}}$

$\dot{\mathrm{V}}_{\mathrm{rv}}=\mathrm{Q}_{\mathrm{tc}}-\mathrm{Q}_{\mathrm{pv}}$

$\dot{\mathrm{V}}_{\mathrm{pa}}=\mathrm{Q}_{\mathrm{pv}}-\mathrm{Q}_{\mathrm{pul}}$

$\dot{\mathrm{V}}_{\mathrm{pu}}=\mathrm{Q}_{\mathrm{pul}}-\mathrm{Q}_{\mathrm{mt}}$

$\mathrm{SBV}=\mathrm{V}_{\mathrm{lv}}+\mathrm{V}_{\mathrm{ao}}+\mathrm{V}_{\mathrm{vc}}+\mathrm{V}_{\mathrm{rV}}+\mathrm{V}_{\mathrm{pa}}+\mathrm{V}_{\mathrm{pu}}$

$S V=\int_{0}^{T} Q d t$

$$
\begin{aligned}
& Q_{m t}=\frac{r\left(P_{p u}-P_{l v}\right)}{R_{m t}} \\
& Q_{a v}=\frac{r\left(P_{l v}-P_{a o}\right)}{R_{a v}} \\
& Q_{s y s}=\frac{P_{a o}-P_{v c}}{R_{s y s}} \\
& Q_{t c}=\frac{r\left(P_{v c}-P_{r v}\right)}{R_{t c}} \\
& Q_{p v}=\frac{r\left(P_{r v}-P_{p a}\right)}{R_{p v}} \\
& Q_{p u l}=\frac{P_{p a}-P_{p u}}{R_{p u l}}
\end{aligned}
$$




\section{During the whole cardiac cycle}

$\dot{\mathrm{V}}_{\mathrm{lv}}=\mathrm{Q}_{\mathrm{mt}}-\mathrm{Q}_{\mathrm{av}}$

$\dot{\mathrm{V}}_{\mathrm{ao}}=\mathrm{Q}_{\mathrm{av}}-\mathrm{Q}_{\mathrm{sys}}$

$\dot{\mathrm{V}}_{\mathrm{vc}}=\mathrm{Q}_{\mathrm{sys}}-\mathrm{Q}_{\mathrm{tc}}$

$\dot{\mathrm{V}}_{\mathrm{rv}}=\mathrm{Q}_{\mathrm{tc}}-\mathrm{Q}_{\mathrm{pv}}$

$\dot{\mathrm{V}}_{\mathrm{pa}}=\mathrm{Q}_{\mathrm{pv}}-\mathrm{Q}_{\mathrm{pul}}$

$\dot{\mathrm{V}}_{\mathrm{pu}}=\mathrm{Q}_{\mathrm{pul}}-\mathrm{Q}_{\mathrm{mt}}$

$\mathrm{SBV}=\mathrm{V}_{\mathrm{lv}}+\mathrm{V}_{\mathrm{ao}}+\mathrm{V}_{\mathrm{vc}}+\mathrm{V}_{\mathrm{rV}}+\mathrm{V}_{\mathrm{pa}}+\mathrm{V}_{\mathrm{pu}}$

$S V=\int_{0}^{T} Q d t$

$$
\begin{aligned}
& Q_{m t}=\frac{r\left(P_{p u}-P_{l v}\right)}{R_{m t}} \\
& Q_{a v}=\frac{r\left(P_{l v}-P_{a o}\right)}{R_{a v}} \\
& Q_{s y s}=\frac{P_{a o}-P_{v c}}{R_{s y s}} \\
& Q_{t c}=\frac{r\left(P_{v c}-P_{r v}\right)}{R_{t c}} \\
& Q_{p v}=\frac{r\left(P_{r v}-P_{p a}\right)}{R_{p v}} \\
& Q_{p u l}=\frac{P_{p a}-P_{p u}}{R_{p u l}}
\end{aligned}
$$




\section{During the whole cardiac cycle}

$S V=\int_{0}^{T} Q d t$

$$
Q_{p u l}=\frac{P_{p a}-P_{p u}}{R_{p u l}}
$$




\section{During the whole cardiac cycle}

$\dot{\mathrm{V}}_{\mathrm{lv}}=\mathrm{Q}_{\mathrm{mt}}-\mathrm{Q}_{\mathrm{av}}$

$\dot{\mathrm{V}}_{\mathrm{ao}}=\mathrm{Q}_{\mathrm{av}}-\mathrm{Q}_{\mathrm{sys}}$

$\dot{\mathrm{V}}_{\mathrm{vc}}=\mathrm{Q}_{\mathrm{sys}}-\mathrm{Q}_{\mathrm{tc}}$

$\dot{\mathrm{V}}_{\mathrm{rv}}=\mathrm{Q}_{\mathrm{tc}}-\mathrm{Q}_{\mathrm{pv}}$

$\dot{\mathrm{V}}_{\mathrm{pa}}=\mathrm{Q}_{\mathrm{pv}}-\mathrm{Q}_{\mathrm{pul}}$

$\dot{\mathrm{V}}_{\mathrm{pu}}=\mathrm{Q}_{\mathrm{pul}}-\mathrm{Q}_{\mathrm{mt}}$

$\mathrm{SBV}=\mathrm{V}_{\mathrm{lv}}+\mathrm{V}_{\mathrm{ao}}+\mathrm{V}_{\mathrm{vc}}+\mathrm{V}_{\mathrm{rV}}+\mathrm{V}_{\mathrm{pa}}+\mathrm{V}_{\mathrm{pu}}$

$S V=\int_{0}^{T} Q d t$

$$
\begin{aligned}
& Q_{m t}=\frac{r\left(P_{p u}-P_{l v}\right)}{R_{m t}} \\
& Q_{a v}=\frac{r\left(P_{l v}-P_{a o}\right)}{R_{a v}} \\
& Q_{s y s}=\frac{P_{a o}-P_{v c}}{R_{s y s}} \\
& Q_{t c}=\frac{r\left(P_{v c}-P_{r v}\right)}{R_{t c}} \\
& Q_{p v}=\frac{r\left(P_{r v}-P_{p a}\right)}{R_{p v}} \\
& Q_{p u l}=\frac{P_{p a}-P_{p u}}{R_{p u l}}
\end{aligned}
$$




\section{During the whole cardiac cycle}

$\dot{\mathrm{V}}_{\mathrm{lv}}=\mathrm{Q}_{\mathrm{mt}}-\mathrm{Q}_{\mathrm{av}}$

$\dot{\mathrm{V}}_{\mathrm{ao}}=\mathrm{Q}_{\mathrm{av}}-\mathrm{Q}_{\mathrm{sys}}$

$\dot{\mathrm{V}}_{\mathrm{vc}}=\mathrm{Q}_{\mathrm{sys}}-\mathrm{Q}_{\mathrm{tc}}$

$\dot{\mathrm{V}}_{\mathrm{rv}}=\mathrm{Q}_{\mathrm{tc}}-\mathrm{Q}_{\mathrm{pv}}$

$\dot{\mathrm{V}}_{\mathrm{pa}}=\mathrm{Q}_{\mathrm{pv}}-\mathrm{Q}_{\mathrm{pul}}$

$\dot{\mathrm{V}}_{\mathrm{pu}}=\mathrm{Q}_{\mathrm{pul}}-\mathrm{Q}_{\mathrm{mt}}$

$\mathrm{SBV}=\mathrm{V}_{\mathrm{lv}}+\mathrm{V}_{\mathrm{ao}}+\mathrm{V}_{\mathrm{vc}}+\mathrm{V}_{\mathrm{rV}}+\mathrm{V}_{\mathrm{pa}}+\mathrm{V}_{\mathrm{pu}}$

$S V=\int_{0}^{T} Q d t$

$$
\begin{aligned}
& Q_{m t}=\frac{r\left(P_{p u}-P_{l v}\right)}{R_{m t}} \\
& Q_{a v}=\frac{r\left(P_{l v}-P_{a o}\right)}{R_{a v}} \\
& Q_{s y s}=\frac{P_{a o}-P_{v c}}{R_{s y s}} \\
& Q_{t c}=\frac{r\left(P_{v c}-P_{r v}\right)}{R_{t c}} \\
& Q_{p v}=\frac{r\left(P_{r v}-P_{p a}\right)}{R_{p v}} \\
& Q_{p u l}=\frac{P_{p a}-P_{p u}}{R_{p u l}}
\end{aligned}
$$


At the beginning of systole: $P_{l v, B S}=P_{a o, B S}$

$\dot{\mathrm{V}}_{\mathrm{lv}}=\mathrm{Q}_{\mathrm{mt}}-\mathrm{Q}_{\mathrm{av}}$

$\dot{\mathrm{V}}_{\mathrm{ao}}=\mathrm{Q}_{\mathrm{av}}-\mathrm{Q}_{\mathrm{sys}}$

$\dot{V}_{\mathrm{vc}}=\mathrm{Q}_{\mathrm{sys}}-\mathrm{Q}_{\mathrm{tc}}$

$\dot{V}_{\mathrm{rv}}=\mathrm{Q}_{\mathrm{tc}}-\mathrm{Q}_{\mathrm{pv}}$

$\dot{V}_{\mathrm{pa}}=\mathrm{Q}_{\mathrm{pv}}-\mathrm{Q}_{\mathrm{pul}}$

$\dot{V}_{\mathrm{pu}}=\mathrm{Q}_{\mathrm{pul}}-\mathrm{Q}_{\mathrm{mt}}$

$S B V=V_{l v}+V_{a o}+V_{v c}+V_{r v}+V_{p a}+V_{p u}$

$S V=\int_{0}^{T} Q d t$

$$
\begin{aligned}
& Q_{m t}=\frac{r\left(P_{p u}-P_{l v}\right)}{R_{m t}} \\
& Q_{a v}=\frac{r\left(P_{l v}-P_{a o}\right)}{R_{a v}} \\
& Q_{s y s}=\frac{P_{a o}-P_{v c}}{R_{s y s}} \\
& Q_{t c}=\frac{r\left(P_{v c}-P_{r v}\right)}{R_{t c}} \\
& Q_{p v}=\frac{r\left(P_{r v}-P_{p a}\right)}{R_{p v}} \\
& Q_{p u l}=\frac{P_{p a}-P_{p u}}{R_{p u l}}
\end{aligned}
$$


At the beginning of systole: $P_{l v, B S}=P_{a o, B S}$

$$
P_{l v}=E_{l v} \cdot e_{l v} \cdot V_{l v}
$$


At the beginning of systole: $P_{l v, B S}=P_{a o, B S}$

$$
P_{l v, B S}=E_{l v} \cdot e_{l v, B S} \cdot V_{l v, B S}
$$


At the beginning of systole: $P_{l v, B S}=P_{a o, B S}$

$$
P_{a o, B S}=E_{l v} \cdot e_{l v, B S} \cdot V_{l v, B S}
$$


At the beginning of systole: $P_{l v, B S}=P_{a o, B S}$

$$
V_{\mathrm{lv}, B S}=\frac{P_{a o, B S}}{E_{l v} \cdot e_{l v, B S}}
$$


At the end of systole: $P_{\mathrm{IV}, E S}=P_{a o, E S}$

$$
V_{I v, B S}=\frac{P_{a o, B S}}{E_{I v} \cdot e_{I v, B S}} \quad V_{I v, E S}=\frac{P_{a o, E S}}{E_{I v} \cdot e_{I v, E S}}
$$




\section{At the end of systole: $P_{I V, E S}=P_{a o, E S}$}

$$
V_{I V, B S}=\frac{P_{a o, B S}}{E_{I V} \cdot e_{I V, B S}} \quad V_{I V, E S}=\frac{P_{a o, E S}}{E_{I V} \cdot e_{I V, E S}}
$$

$$
S V=V_{I V, B S}-V_{I V, E S}
$$




\section{At the end of systole: $P_{\mathrm{IV}, E S}=P_{a O, E S}$}

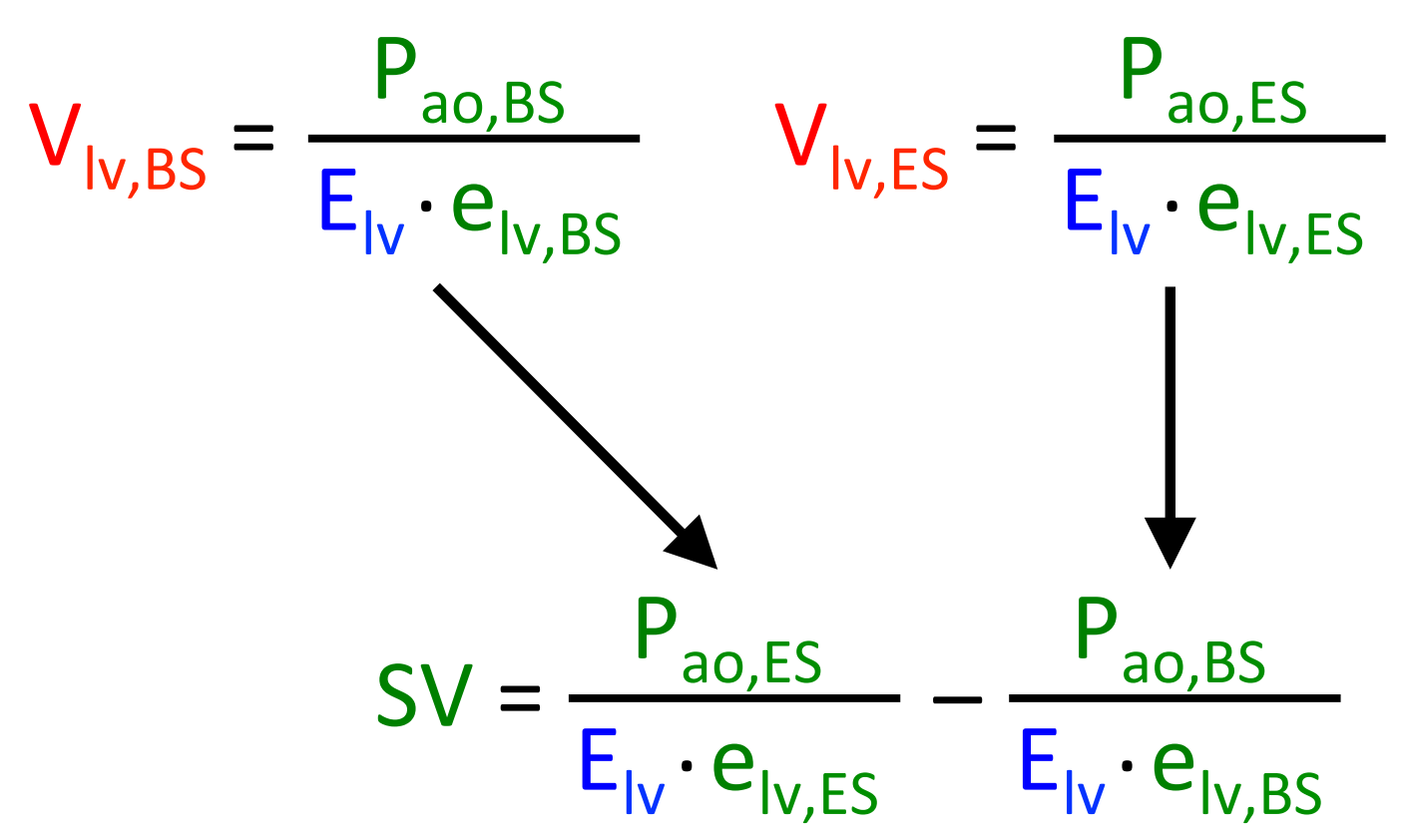




\section{At the end of systole: $P_{I V, E S}=P_{a o, E S}$}

$$
V_{l v, B S}=\frac{P_{a o, B S}}{E_{l v} \cdot e_{l v, B S}} \quad V_{l v, E S}=\frac{P_{a o, E S}}{E_{l v} \cdot e_{l v, E S}}
$$

$$
E_{l v}=\frac{P_{a o, E S}}{S V \cdot e_{I V, E S}}-\frac{P_{a o, B S}}{S V \cdot e_{I V, B S}}
$$




\section{At the end of systole: $P_{I V, E S}=P_{a o, E S}$}

$\dot{\mathrm{V}}_{\mathrm{lv}}=\mathrm{Q}_{\mathrm{mt}}-\mathrm{Q}_{\mathrm{av}}$

$\dot{\mathrm{V}}_{\mathrm{ao}}=\mathrm{Q}_{\mathrm{av}}-\mathrm{Q}_{\mathrm{sys}}$

$\dot{\mathrm{V}}_{\mathrm{vc}}=\mathrm{Q}_{\mathrm{sys}}-\mathrm{Q}_{\mathrm{tc}}$

$\dot{\mathrm{V}}_{\mathrm{rv}}=\mathrm{Q}_{\mathrm{tc}}-\mathrm{Q}_{\mathrm{pv}}$

$\dot{\mathrm{V}}_{\mathrm{pa}}=\mathrm{Q}_{\mathrm{pv}}-\mathrm{Q}_{\mathrm{pul}}$

$\dot{\mathrm{V}}_{\mathrm{pu}}=\mathrm{Q}_{\text {pul }}-\mathrm{Q}_{\mathrm{mt}}$

$\mathrm{SBV}=\mathrm{V}_{\mathrm{lv}}+\mathrm{V}_{\mathrm{ao}}+\mathrm{V}_{\mathrm{vc}}+\mathrm{V}_{\mathrm{rV}}+\mathrm{V}_{\mathrm{pa}}+\mathrm{V}_{\mathrm{pu}}$

$S V=\int_{0}^{T} Q d t$

$$
\begin{aligned}
& Q_{m t}=\frac{r\left(P_{p u}-P_{l v}\right)}{R_{m t}} \\
& Q_{a v}=\frac{r\left(P_{\mathrm{Il}}-P_{a 0}\right)}{R_{a v}} \\
& Q_{s y s}=\frac{P_{a o}-P_{v c}}{R_{s y s}} \\
& Q_{t c}=\frac{r\left(P_{v v}-P_{r v}\right)}{R_{t c}} \\
& Q_{p v}=\frac{r\left(P_{r v}-P_{p a}\right)}{R_{p v}} \\
& Q_{p u l}=\frac{P_{p a}-P_{p u}}{R_{p u l}}
\end{aligned}
$$




\section{At the end of systole: $P_{I V, E S}=P_{a o, E S}$}

$\dot{\mathrm{V}}_{\mathrm{lv}}=\mathrm{Q}_{\mathrm{mt}}-\mathrm{Q}_{\mathrm{av}}$

$\dot{\mathrm{V}}_{\mathrm{ao}}=\mathrm{Q}_{\mathrm{av}}-\mathrm{Q}_{\text {sys }}$

$\dot{\mathrm{V}}_{\mathrm{vc}}=\mathrm{Q}_{\mathrm{sys}}-\mathrm{Q}_{\mathrm{tc}}$

$\dot{\mathrm{V}}_{\mathrm{rv}}=\mathrm{Q}_{\mathrm{tc}}-\mathrm{Q}_{\mathrm{pv}}$

$\dot{\mathrm{V}}_{\mathrm{pa}}=\mathrm{Q}_{\mathrm{pv}}-\mathrm{Q}_{\mathrm{pul}}$

$\dot{\mathrm{V}}_{\mathrm{pu}}=\mathrm{Q}_{\mathrm{pul}}-\mathrm{Q}_{\mathrm{mt}}$

$\mathrm{SBV}=\mathrm{V}_{\mathrm{lv}}+\mathrm{V}_{\mathrm{ao}}+\mathrm{V}_{\mathrm{vc}}+\mathrm{V}_{\mathrm{rV}}+\mathrm{V}_{\mathrm{pa}}+\mathrm{V}_{\mathrm{pu}}$

$S V=\int_{0}^{T} Q d t$

$$
\begin{aligned}
& Q_{m t}=\frac{r\left(P_{p u}-P_{l v}\right)}{R_{m t}} \\
& Q_{a v}=\frac{r\left(P_{\mathrm{Il}}-P_{a 0}\right)}{R_{a v}} \\
& Q_{s y s}=\frac{P_{a o}-P_{v c}}{R_{s y s}} \\
& Q_{t c}=\frac{r\left(P_{v v}-P_{r v}\right)}{R_{t c}} \\
& Q_{p v}=\frac{r\left(P_{r v}-P_{p a}\right)}{R_{p v}} \\
& Q_{p u l}=\frac{P_{p a}-P_{p u}}{R_{p u l}}
\end{aligned}
$$




\section{At the end of systole: $P_{r v, E S}=P_{p a, E S}$}

$\dot{\mathrm{V}}_{\mathrm{lv}}=\mathrm{Q}_{\mathrm{mt}}-\mathrm{Q}_{\mathrm{av}}$

$\dot{\mathrm{V}}_{\mathrm{ao}}=\mathrm{Q}_{\mathrm{av}}-\mathrm{Q}_{\mathrm{sys}}$

$\dot{\mathrm{V}}_{\mathrm{vc}}=\mathrm{Q}_{\mathrm{sys}}-\mathrm{Q}_{\mathrm{tc}}$

$\dot{\mathrm{V}}_{\mathrm{rv}}=\mathrm{Q}_{\mathrm{tc}}-\mathrm{Q}_{\mathrm{pv}}$

$\dot{\mathrm{V}}_{\mathrm{pa}}=\mathrm{Q}_{\mathrm{pv}}-\mathrm{Q}_{\mathrm{pul}}$

$\dot{\mathrm{V}}_{\mathrm{pu}}=\mathrm{Q}_{\mathrm{pul}}-\mathrm{Q}_{\mathrm{mt}}$

$\mathrm{SBV}=\mathrm{V}_{\mathrm{lv}}+\mathrm{V}_{\mathrm{ao}}+\mathrm{V}_{\mathrm{vc}}+\mathrm{V}_{\mathrm{rV}}+\mathrm{V}_{\mathrm{pa}}+\mathrm{V}_{\mathrm{pu}}$

$S V=\int_{0}^{T} Q d t$

$$
\begin{aligned}
& Q_{m t}=\frac{r\left(P_{p u}-P_{l v}\right)}{R_{m t}} \\
& Q_{a v}=\frac{r\left(P_{\mathrm{Il}}-P_{a 0}\right)}{R_{a v}} \\
& Q_{s y s}=\frac{P_{a o}-P_{v c}}{R_{s y s}} \\
& Q_{t c}=\frac{r\left(P_{v v}-P_{r v}\right)}{R_{t c}} \\
& Q_{p v}=\frac{r\left(P_{r v}-P_{p a}\right)}{R_{p v}} \\
& Q_{p u l}=\frac{P_{p a}-P_{p u}}{R_{p u l}}
\end{aligned}
$$




\section{During cardiac ejection: $P_{r v}>P_{v c}$}

$\dot{\mathrm{V}}_{\mathrm{lv}}=\mathrm{Q}_{\mathrm{mt}}-\mathrm{Q}_{\mathrm{av}}$

$\dot{\mathrm{V}}_{\mathrm{ao}}=\mathrm{Q}_{\mathrm{av}}-\mathrm{Q}_{\mathrm{sys}}$

$\dot{\mathrm{V}}_{\mathrm{vc}}=\mathrm{Q}_{\mathrm{sys}}-\mathrm{Q}_{\mathrm{tc}}$

$\dot{\mathrm{V}}_{\mathrm{rv}}=\mathrm{Q}_{\mathrm{tc}}-\mathrm{Q}_{\mathrm{pv}}$

$\dot{\mathrm{V}}_{\mathrm{pa}}=\mathrm{Q}_{\mathrm{pv}}-\mathrm{Q}_{\mathrm{pul}}$

$\dot{\mathrm{V}}_{\mathrm{pu}}=\mathrm{Q}_{\mathrm{pul}}-\mathrm{Q}_{\mathrm{mt}}$

$\mathrm{SBV}=\mathrm{V}_{\mathrm{lv}}+\mathrm{V}_{\mathrm{ao}}+\mathrm{V}_{\mathrm{vc}}+\mathrm{V}_{\mathrm{rV}}+\mathrm{V}_{\mathrm{pa}}+\mathrm{V}_{\mathrm{pu}}$

$S V=\int_{0}^{T} Q d t$

$$
\begin{aligned}
& Q_{m t}=\frac{r\left(P_{p u}-P_{l v}\right)}{R_{m t}} \\
& Q_{a v}=\frac{r\left(P_{l v}-P_{a o}\right)}{R_{a v}} \\
& Q_{s y s}=\frac{P_{a o}-P_{v c}}{R_{s y s}} \\
& Q_{t c}=\frac{r\left(P_{v c}-P_{r v}\right)}{R_{t c}} \\
& Q_{p v}=\frac{r\left(P_{r v}-P_{p a}\right)}{R_{p v}} \\
& Q_{p u l}=\frac{P_{p a}-P_{p u}}{R_{p u l}}
\end{aligned}
$$




\section{During cardiac ejection: $P_{r v}>P_{v c}$}

$\dot{\mathrm{V}}_{\mathrm{vc}}=\mathrm{Q}_{\mathrm{sys}}-\mathrm{Q}_{\mathrm{tc}}$

$$
P_{v c}=E_{v c} \cdot V_{v c}
$$

$$
\begin{aligned}
& Q_{s y s}=\frac{P_{a o}-P_{v c}}{R_{s y s}} \\
& Q_{t c}=\frac{r\left(P_{v c}-P_{r v}\right)}{R_{t c}}
\end{aligned}
$$




\section{During cardiac ejection: $P_{r v}>P_{v c}$}

$\dot{\mathrm{V}}_{\mathrm{vc}}=\mathrm{Q}_{\mathrm{sys}}-\mathrm{Q}_{\mathrm{tc}}$

$$
P_{v c}=E_{v c} \cdot V_{v c}
$$$$
\begin{aligned}
& Q_{\text {sys }}=\frac{P_{a 0}-P_{v c}}{R_{s y s}} \\
& Q_{t c}=0
\end{aligned}
$$ 


\section{During cardiac ejection: $P_{r v}>P_{v c}$}

$\dot{\mathrm{V}}_{\mathrm{vc}}=\mathrm{Q}_{\mathrm{sys}}-\mathrm{Q}_{\mathrm{tc}}$

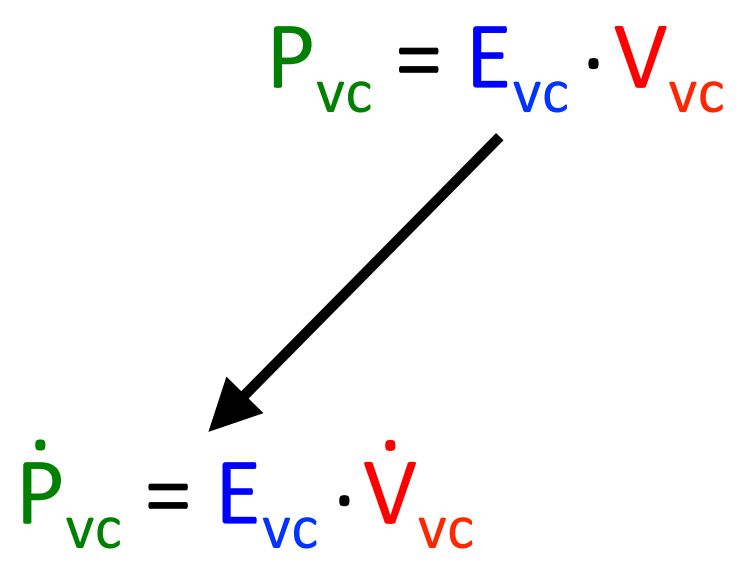

$Q_{\text {sys }}=\frac{P_{\text {ao }}-P_{v c}}{R_{s y s}}$
$Q_{t c}=0$ 


\section{During cardiac ejection: $P_{r v}>P_{v c}$}

$\dot{\mathrm{V}}_{\mathrm{vc}}=\mathrm{Q}_{\mathrm{sys}}-\mathrm{Q}_{\mathrm{tc}}$

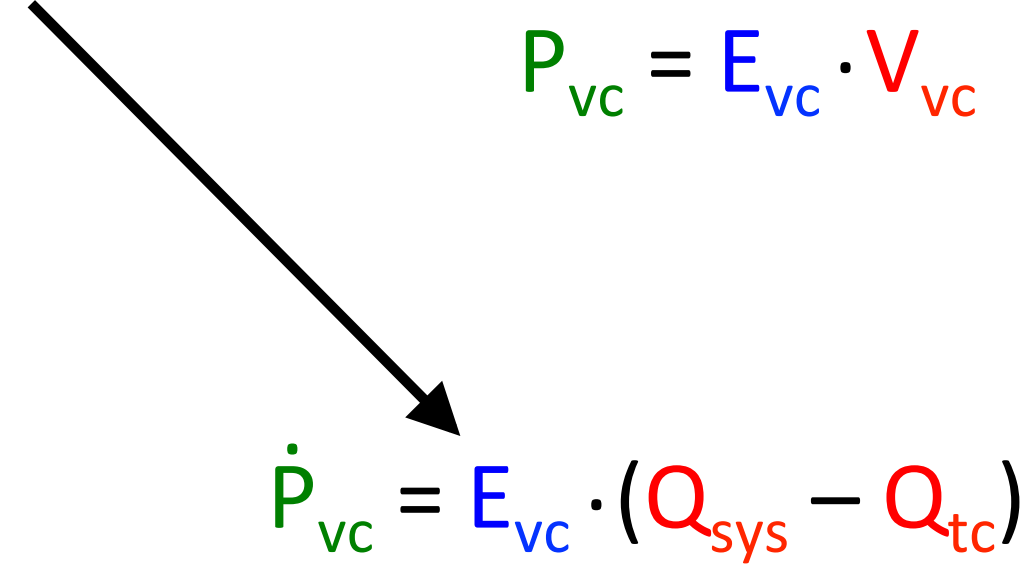

$$
\begin{aligned}
& \mathrm{Q}_{\mathrm{sys}}=\frac{\mathrm{P}_{\mathrm{ao}}-\mathrm{P}_{\mathrm{vc}}}{\mathrm{R}_{\mathrm{sys}}} \\
& \mathrm{Q}_{\mathrm{tc}}=0
\end{aligned}
$$




\section{During cardiac ejection: $P_{r v}>P_{v c}$}

$\dot{\mathrm{V}}_{\mathrm{vc}}=\mathrm{Q}_{\mathrm{sys}}-\mathrm{Q}_{\mathrm{tc}}$

$$
\dot{P}_{\mathrm{vc}}=\mathrm{E}_{\mathrm{vc}} \cdot \frac{\mathrm{P}_{\mathrm{ac}}-\mathrm{P}_{\mathrm{vc}}=\mathrm{E}_{\mathrm{vc}} \cdot \mathrm{V}_{\mathrm{vc}}}{\mathrm{R}_{\mathrm{sys}}} \mathrm{Q}_{\mathrm{tc}}=0
$$




\section{During cardiac ejection: $P_{r v}>P_{v c}$}

$\dot{\mathrm{V}}_{\mathrm{vc}}=\mathrm{Q}_{\mathrm{sys}}-\mathrm{Q}_{\mathrm{tc}}$

$$
\begin{aligned}
& Q_{s y s}=\frac{P_{a 0}-P_{v c}}{R_{s y s}} \\
& Q_{t c}=0
\end{aligned}
$$$$
\frac{P_{v c} \cdot R_{s y s}}{P_{a o}-P_{v c}}=E_{v c}
$$ 


\section{During cardiac ejection: $P_{r v}>P_{v c}$}

$\dot{\mathrm{V}}_{\mathrm{lv}}=\mathrm{Q}_{\mathrm{mt}}-\mathrm{Q}_{\mathrm{av}}$

$\dot{\mathrm{V}}_{\mathrm{ao}}=\mathrm{Q}_{\mathrm{av}}-\mathrm{Q}_{\mathrm{sys}}$

$\dot{\mathrm{V}}_{\mathrm{vc}}=\mathrm{Q}_{\mathrm{sys}}-\mathrm{Q}_{\mathrm{tc}}$

$\dot{\mathrm{V}}_{\mathrm{rv}}=\mathrm{Q}_{\mathrm{tc}}-\mathrm{Q}_{\mathrm{pv}}$

$\dot{\mathrm{V}}_{\mathrm{pa}}=\mathrm{Q}_{\mathrm{pv}}-\mathrm{Q}_{\mathrm{pul}}$

$\dot{\mathrm{V}}_{\mathrm{pu}}=\mathrm{Q}_{\mathrm{pul}}-\mathrm{Q}_{\mathrm{mt}}$

$\mathrm{SBV}=\mathrm{V}_{\mathrm{lv}}+\mathrm{V}_{\mathrm{ao}}+\mathrm{V}_{\mathrm{vc}}+\mathrm{V}_{\mathrm{rV}}+\mathrm{V}_{\mathrm{pa}}+\mathrm{V}_{\mathrm{pu}}$

$S V=\int_{0}^{T} Q d t$

$$
\begin{aligned}
& Q_{m t}=\frac{r\left(P_{p u}-P_{l v}\right)}{R_{m t}} \\
& Q_{a v}=\frac{r\left(P_{l v}-P_{a o}\right)}{R_{a v}} \\
& Q_{s y s}=\frac{P_{a o}-P_{v c}}{R_{s y s}} \\
& Q_{t c}=\frac{r\left(P_{v c}-P_{r v}\right)}{R_{t c}} \\
& Q_{p v}=\frac{r\left(P_{r v}-P_{p a}\right)}{R_{p v}} \\
& Q_{p u l}=\frac{P_{p a}-P_{p u}}{R_{p u l}}
\end{aligned}
$$




\section{During cardiac ejection: $P_{r v}>P_{v c}$}

$\dot{\mathrm{V}}_{\mathrm{lv}}=\mathrm{Q}_{\mathrm{mt}}-\mathrm{Q}_{\mathrm{av}}$

$\dot{\mathrm{V}}_{\mathrm{ao}}=\mathrm{Q}_{\mathrm{av}}-\mathrm{Q}_{\mathrm{sys}}$

$\dot{\mathrm{V}}_{\mathrm{vc}}=\mathrm{Q}_{\mathrm{sys}}-\mathrm{Q}_{\mathrm{tc}}$

$\dot{\mathrm{V}}_{\mathrm{rv}}=\mathrm{Q}_{\mathrm{tc}}-\mathrm{Q}_{\mathrm{pv}}$

$\dot{\mathrm{V}}_{\mathrm{pa}}=\mathrm{Q}_{\mathrm{pv}}-\mathrm{Q}_{\mathrm{pul}}$

$\dot{\mathrm{V}}_{\mathrm{pu}}=\mathrm{Q}_{\mathrm{pul}}-\mathrm{Q}_{\mathrm{mt}}$

$\mathrm{SBV}=\mathrm{V}_{\mathrm{lv}}+\mathrm{V}_{\mathrm{ao}}+\mathrm{V}_{\mathrm{vc}}+\mathrm{V}_{\mathrm{rV}}+\mathrm{V}_{\mathrm{pa}}+\mathrm{V}_{\mathrm{pu}}$

$S V=\int_{0}^{T} Q d t$

$$
\begin{aligned}
& Q_{m t}=\frac{r\left(P_{p u}-P_{l v}\right)}{R_{m t}} \\
& Q_{a v}=\frac{r\left(P_{l v}-P_{a o}\right)}{R_{a v}} \\
& Q_{s y s}=\frac{P_{a o}-P_{v c}}{R_{s y s}} \\
& Q_{t c}=\frac{r\left(P_{v c}-P_{r v}\right)}{R_{t c}} \\
& Q_{p v}=\frac{r\left(P_{r v}-P_{p a}\right)}{R_{p v}} \\
& Q_{p u l}=\frac{P_{p a}-P_{p u}}{R_{p u l}}
\end{aligned}
$$




\section{During cardiac ejection: $P_{\mathrm{lv}}>P_{p u}$}

$\dot{\mathrm{V}}_{\mathrm{lv}}=\mathrm{Q}_{\mathrm{mt}}-\mathrm{Q}_{\mathrm{av}}$

$\dot{\mathrm{V}}_{\mathrm{ao}}=\mathrm{Q}_{\mathrm{av}}-\mathrm{Q}_{\mathrm{sys}}$

$\dot{\mathrm{V}}_{\mathrm{vc}}=\mathrm{Q}_{\mathrm{sys}}-\mathrm{Q}_{\mathrm{tc}}$

$\dot{\mathrm{V}}_{\mathrm{rv}}=\mathrm{Q}_{\mathrm{tc}}-\mathrm{Q}_{\mathrm{pv}}$

$\dot{\mathrm{V}}_{\mathrm{pa}}=\mathrm{Q}_{\mathrm{pv}}-\mathrm{Q}_{\mathrm{pul}}$

$\dot{\mathrm{V}}_{\mathrm{pu}}=\mathrm{Q}_{\mathrm{pul}}-\mathrm{Q}_{\mathrm{mt}}$

$S B V=V_{l v}+V_{a o}+V_{v c}+V_{r V}+V_{p a}+V_{p u}$

$S V=\int_{0}^{T} Q d t$

$$
\begin{aligned}
& Q_{m t}=\frac{r\left(P_{p u}-P_{l v}\right)}{R_{m t}} \\
& Q_{a v}=\frac{r\left(P_{l v}-P_{a o}\right)}{R_{a v}} \\
& Q_{s y s}=\frac{P_{a o}-P_{v c}}{R_{s y s}} \\
& Q_{t c}=\frac{r\left(P_{v c}-P_{r v}\right)}{R_{t c}} \\
& Q_{p v}=\frac{r\left(P_{r v}-P_{p a}\right)}{R_{p v}} \\
& Q_{p u l}=\frac{P_{p a}-P_{p u}}{R_{p u l}}
\end{aligned}
$$




\section{During cardiac filling: $P_{a o}>P_{l v}$}

$\dot{\mathrm{V}}_{\mathrm{lv}}=\mathrm{Q}_{\mathrm{mt}}-\mathrm{Q}_{\mathrm{av}}$

$\dot{\mathrm{V}}_{\mathrm{ao}}=\mathrm{Q}_{\mathrm{av}}-\mathrm{Q}_{\mathrm{sys}}$

$\dot{\mathrm{V}}_{\mathrm{vc}}=\mathrm{Q}_{\mathrm{sys}}-\mathrm{Q}_{\mathrm{tc}}$

$\dot{\mathrm{V}}_{\mathrm{rv}}=\mathrm{Q}_{\mathrm{tc}}-\mathrm{Q}_{\mathrm{pv}}$

$\dot{\mathrm{V}}_{\mathrm{pa}}=\mathrm{Q}_{\mathrm{pv}}-\mathrm{Q}_{\mathrm{pul}}$

$\dot{\mathrm{V}}_{\mathrm{pu}}=\mathrm{Q}_{\mathrm{pul}}-\mathrm{Q}_{\mathrm{mt}}$

$S B V=V_{l v}+V_{a o}+V_{v c}+V_{r V}+V_{p a}+V_{p u}$

$S V=\int_{0}^{T} Q d t$

$$
\begin{aligned}
& Q_{m t}=\frac{r\left(P_{p u}-P_{l v}\right)}{R_{m t}} \\
& Q_{a v}=\frac{r\left(P_{l v}-P_{a o}\right)}{R_{a v}} \\
& Q_{s y s}=\frac{P_{a o}-P_{v c}}{R_{s y s}} \\
& Q_{t c}=\frac{r\left(P_{v c}-P_{r v}\right)}{R_{t c}} \\
& Q_{p v}=\frac{r\left(P_{r v}-P_{p a}\right)}{R_{p v}} \\
& Q_{p u l}=\frac{P_{p a}-P_{p u}}{R_{p u l}}
\end{aligned}
$$




\section{During cardiac filling: $P_{a o}>P_{l v}$}

$\dot{\mathrm{V}}_{\mathrm{ao}}=\mathrm{Q}_{\mathrm{av}}-\mathrm{Q}_{\mathrm{sys}}$

$$
\mathrm{P}_{\mathrm{ao}}=\mathrm{E}_{\mathrm{ao}} \cdot \mathrm{V}_{\mathrm{ao}}
$$

$$
\begin{aligned}
& Q_{a v}=\frac{r\left(P_{l v}-P_{a o}\right)}{R_{a v}} \\
& Q_{s y s}=\frac{P_{a o}-P_{v c}}{R_{s y s}}
\end{aligned}
$$




\section{During cardiac filling: $P_{a o}>P_{l v}$}

$\dot{\mathrm{V}}_{\mathrm{ao}}=\mathrm{Q}_{\mathrm{av}}-\mathrm{Q}_{\mathrm{sys}}$

$$
P_{\text {ao }}=E_{a o} \cdot V_{a o}
$$

$\mathrm{Q}_{\mathrm{av}}=0$

$$
\mathrm{Q}_{\text {sys }}=\frac{\mathrm{P}_{\mathrm{ao}}-\mathrm{P}_{\mathrm{vc}}}{\mathrm{R}_{\mathrm{sys}}}
$$




\section{During cardiac filling: $P_{a o}>P_{l v}$}

$\dot{\mathrm{V}}_{\mathrm{ao}}=\mathrm{Q}_{\mathrm{av}}-\mathrm{Q}_{\mathrm{sys}}$

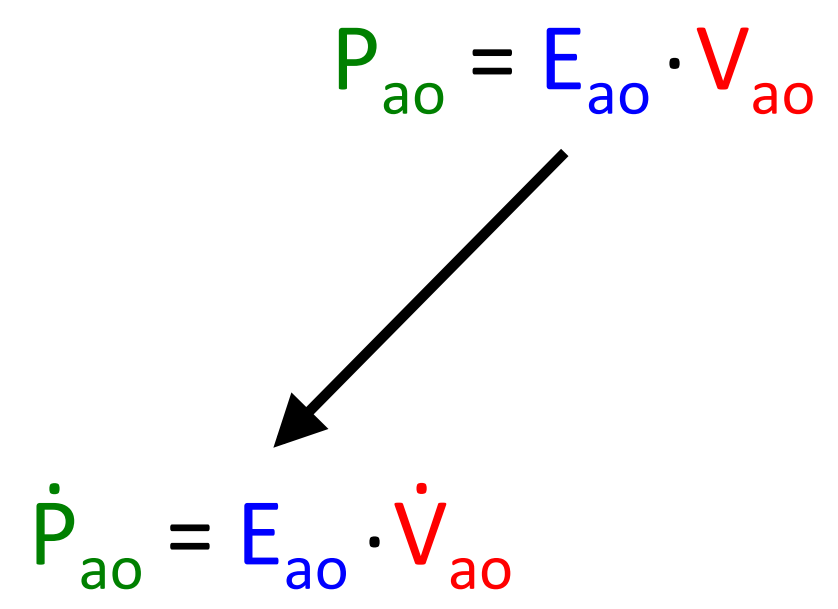

$\mathrm{Q}_{\mathrm{av}}=0$

$\mathrm{Q}_{\text {sys }}=\frac{\mathrm{P}_{\mathrm{ao}}-\mathrm{P}_{\mathrm{vc}}}{\mathrm{R}_{\mathrm{sys}}}$ 


\section{During cardiac filling: $P_{a o}>P_{l v}$}

$\dot{\mathrm{V}}_{\mathrm{ao}}=\mathrm{Q}_{\mathrm{av}}-\mathrm{Q}_{\mathrm{sys}}$

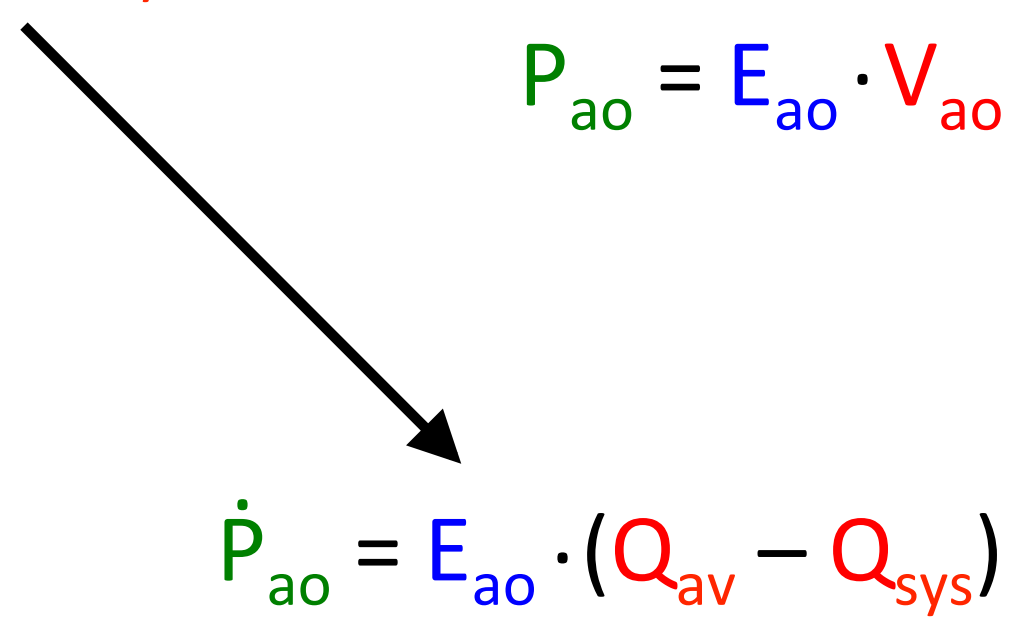

$\mathrm{Q}_{\mathrm{av}}=0$

$\mathrm{Q}_{\text {sys }}=\frac{\mathrm{P}_{\mathrm{ao}}-\mathrm{P}_{\mathrm{vc}}}{\mathrm{R}_{\mathrm{sys}}}$ 


\section{During cardiac filling: $P_{a o}>P_{l v}$}

$\dot{\mathrm{V}}_{\mathrm{ao}}=\mathrm{Q}_{\mathrm{av}}-\mathrm{Q}_{\mathrm{sys}}$

$$
\dot{\mathrm{P}}_{\mathrm{ao}}=-\mathrm{E}_{\mathrm{ao}} \cdot \frac{\mathrm{P}_{\mathrm{ao}}-\mathrm{P}_{\mathrm{vc}}}{\mathrm{R}_{\mathrm{sys}}} \cdot \mathrm{V}_{\mathrm{ao}} \mathrm{Q}_{\mathrm{av}}=0
$$




\section{During cardiac filling: $P_{a o}>P_{l v}$}

$\dot{\mathrm{V}}_{\mathrm{ao}}=\mathrm{Q}_{\mathrm{av}}-\mathrm{Q}_{\mathrm{sys}}$

$$
\begin{array}{r}
P_{\mathrm{ao}}=\mathrm{E}_{\mathrm{ao}} \cdot \mathrm{V}_{\mathrm{ao}} \\
-\frac{\dot{\mathrm{P}}_{\mathrm{ao}} \cdot \mathrm{R}_{\mathrm{sys}}}{\mathrm{P}_{\mathrm{ao}}-\mathrm{P}_{\mathrm{vc}}}=\mathrm{E}_{\mathrm{ao}}
\end{array}
$$$$
\mathrm{Q}_{\mathrm{av}}=0
$$

$$
\mathrm{Q}_{\text {sys }}=\frac{\mathrm{P}_{\mathrm{ao}}-\mathrm{P}_{\mathrm{vc}}}{\mathrm{R}_{\mathrm{sys}}}
$$




\section{During cardiac filling: $P_{a o}>P_{l v}$}

$\dot{\mathrm{V}}_{\mathrm{lv}}=\mathrm{Q}_{\mathrm{mt}}-\mathrm{Q}_{\mathrm{av}}$

$\dot{\mathrm{V}}_{\mathrm{ao}}=\mathrm{Q}_{\mathrm{av}}-\mathrm{Q}_{\mathrm{sys}}$

$\dot{\mathrm{V}}_{\mathrm{vc}}=\mathrm{Q}_{\mathrm{sys}}-\mathrm{Q}_{\mathrm{tc}}$

$\dot{\mathrm{V}}_{\mathrm{rv}}=\mathrm{Q}_{\mathrm{tc}}-\mathrm{Q}_{\mathrm{pv}}$

$\dot{\mathrm{V}}_{\mathrm{pa}}=\mathrm{Q}_{\mathrm{pv}}-\mathrm{Q}_{\mathrm{pul}}$

$\dot{\mathrm{V}}_{\mathrm{pu}}=\mathrm{Q}_{\mathrm{pul}}-\mathrm{Q}_{\mathrm{mt}}$

$S B V=V_{l v}+V_{a o}+V_{v c}+V_{r V}+V_{p a}+V_{p u}$

$S V=\int_{0}^{T} Q d t$

$$
\begin{aligned}
& Q_{m t}=\frac{r\left(P_{p u}-P_{l v}\right)}{R_{m t}} \\
& Q_{a v}=\frac{r\left(P_{l v}-P_{a o}\right)}{R_{a v}} \\
& Q_{s y s}=\frac{P_{a o}-P_{v c}}{R_{s y s}} \\
& Q_{t c}=\frac{r\left(P_{v c}-P_{r v}\right)}{R_{t c}} \\
& Q_{p v}=\frac{r\left(P_{r v}-P_{p a}\right)}{R_{p v}} \\
& Q_{p u l}=\frac{P_{p a}-P_{p u}}{R_{p u l}}
\end{aligned}
$$




\section{During cardiac filling: $P_{a o}>P_{l v}$}

$\dot{\mathrm{V}}_{\mathrm{lv}}=\mathrm{Q}_{\mathrm{mt}}-\mathrm{Q}_{\mathrm{av}}$

$\dot{\mathrm{V}}_{\mathrm{ao}}=\mathrm{Q}_{\mathrm{av}}-\mathrm{Q}_{\mathrm{sys}}$

$\dot{\mathrm{V}}_{\mathrm{vc}}=\mathrm{Q}_{\mathrm{sys}}-\mathrm{Q}_{\mathrm{tc}}$

$\dot{\mathrm{V}}_{\mathrm{rv}}=\mathrm{Q}_{\mathrm{tc}}-\mathrm{Q}_{\mathrm{pv}}$

$\dot{\mathrm{V}}_{\mathrm{pa}}=\mathrm{Q}_{\mathrm{pv}}-\mathrm{Q}_{\mathrm{pul}}$

$\dot{\mathrm{V}}_{\mathrm{pu}}=\mathrm{Q}_{\mathrm{pul}}-\mathrm{Q}_{\mathrm{mt}}$

$S B V=V_{l v}+V_{a o}+V_{v c}+V_{r V}+V_{p a}+V_{p u}$

$S V=\int_{0}^{T} Q d t$

$$
\begin{aligned}
& Q_{m t}=\frac{r\left(P_{p u}-P_{l v}\right)}{R_{m t}} \\
& Q_{a v}=\frac{r\left(P_{l v}-P_{a o}\right)}{R_{a v}} \\
& Q_{s y s}=\frac{P_{a o}-P_{v c}}{R_{s y s}} \\
& Q_{t c}=\frac{r\left(P_{v c}-P_{r v}\right)}{R_{t c}} \\
& Q_{p v}=\frac{r\left(P_{r v}-P_{p a}\right)}{R_{p v}} \\
& Q_{p u l}=\frac{P_{p a}-P_{p u}}{R_{p u l}}
\end{aligned}
$$




\section{During cardiac filling: $P_{v c}>P_{r v}$ and $P_{r v}<P_{p a}$}

$\dot{\mathrm{V}}_{\mathrm{lv}}=\mathrm{Q}_{\mathrm{mt}}-\mathrm{Q}_{\mathrm{av}}$

$\dot{\mathrm{V}}_{\mathrm{ao}}=\mathrm{Q}_{\mathrm{av}}-\mathrm{Q}_{\mathrm{sys}}$

$\dot{\mathrm{V}}_{\mathrm{vc}}=\mathrm{Q}_{\mathrm{sys}}-\mathrm{Q}_{\mathrm{tc}}$

$\dot{\mathrm{V}}_{\mathrm{rv}}=\mathrm{Q}_{\mathrm{tc}}-\mathrm{Q}_{\mathrm{pv}}$

$\dot{\mathrm{V}}_{\mathrm{pa}}=\mathrm{Q}_{\mathrm{pv}}-\mathrm{Q}_{\mathrm{pul}}$

$\dot{\mathrm{V}}_{\mathrm{pu}}=\mathrm{Q}_{\mathrm{pul}}-\mathrm{Q}_{\mathrm{mt}}$

$\mathrm{SBV}=\mathrm{V}_{\mathrm{lv}}+\mathrm{V}_{\mathrm{ao}}+\mathrm{V}_{\mathrm{vc}}+\mathrm{V}_{\mathrm{rV}}+\mathrm{V}_{\mathrm{pa}}+\mathrm{V}_{\mathrm{pu}}$

$S V=\int_{0}^{T} Q d t$

$$
\begin{aligned}
& Q_{m t}=\frac{r\left(P_{p u}-P_{l v}\right)}{R_{m t}} \\
& Q_{a v}=\frac{r\left(P_{l v}-P_{a o}\right)}{R_{a v}} \\
& Q_{s y s}=\frac{P_{a o}-P_{v c}}{R_{s y s}} \\
& Q_{t c}=\frac{r\left(P_{v c}-P_{r v}\right)}{R_{t c}} \\
& Q_{p v}=\frac{r\left(P_{r v}-P_{p a}\right)}{R_{p v}} \\
& Q_{p u l}=\frac{P_{p a}-P_{p u}}{R_{p u l}}
\end{aligned}
$$




\section{During cardiac filling: $P_{v c}>P_{r v}$ and $P_{r v}<P_{p a}$}

$$
\begin{aligned}
& \dot{V}_{v c}=Q_{s y s}-Q_{t c} \\
& \dot{V}_{r v}=Q_{t c}-Q_{p v}
\end{aligned}
$$

$$
\begin{aligned}
& P_{v c}=E_{v c} \cdot V_{v c} \\
& P_{r v}=E_{r v} \cdot e_{r v} \cdot V_{r v}
\end{aligned}
$$

$$
\begin{aligned}
& Q_{s y s}=\frac{P_{a o}-P_{v c}}{R_{s y s}} \\
& Q_{t c}=\frac{r\left(P_{v c}-P_{r v}\right)}{R_{t c}} \\
& Q_{p v}=\frac{r\left(P_{r v}-P_{p a}\right)}{R_{p v}}
\end{aligned}
$$




\section{During cardiac filling: $P_{v c}>P_{r v}$ and $P_{r v}<P_{p a}$}

$$
\begin{aligned}
& \dot{V}_{v c}=Q_{s y s}-Q_{t c} \\
& \dot{V}_{r v}=Q_{t c}-Q_{p v}
\end{aligned}
$$

$$
\begin{aligned}
& P_{v c}=E_{v c} \cdot V_{v c} \\
& P_{r v}=E_{r v} \cdot e_{r v} \cdot V_{r v}
\end{aligned}
$$

$$
\begin{aligned}
& Q_{s y s}=\frac{P_{a o}-P_{v c}}{R_{s y s}} \\
& Q_{t c}=\frac{P_{v c}-P_{r v}}{R_{t c}} \\
& Q_{p v}=0
\end{aligned}
$$




\section{During cardiac filling: $P_{v c}>P_{r v}$ and $P_{r v}<P_{p a}$}

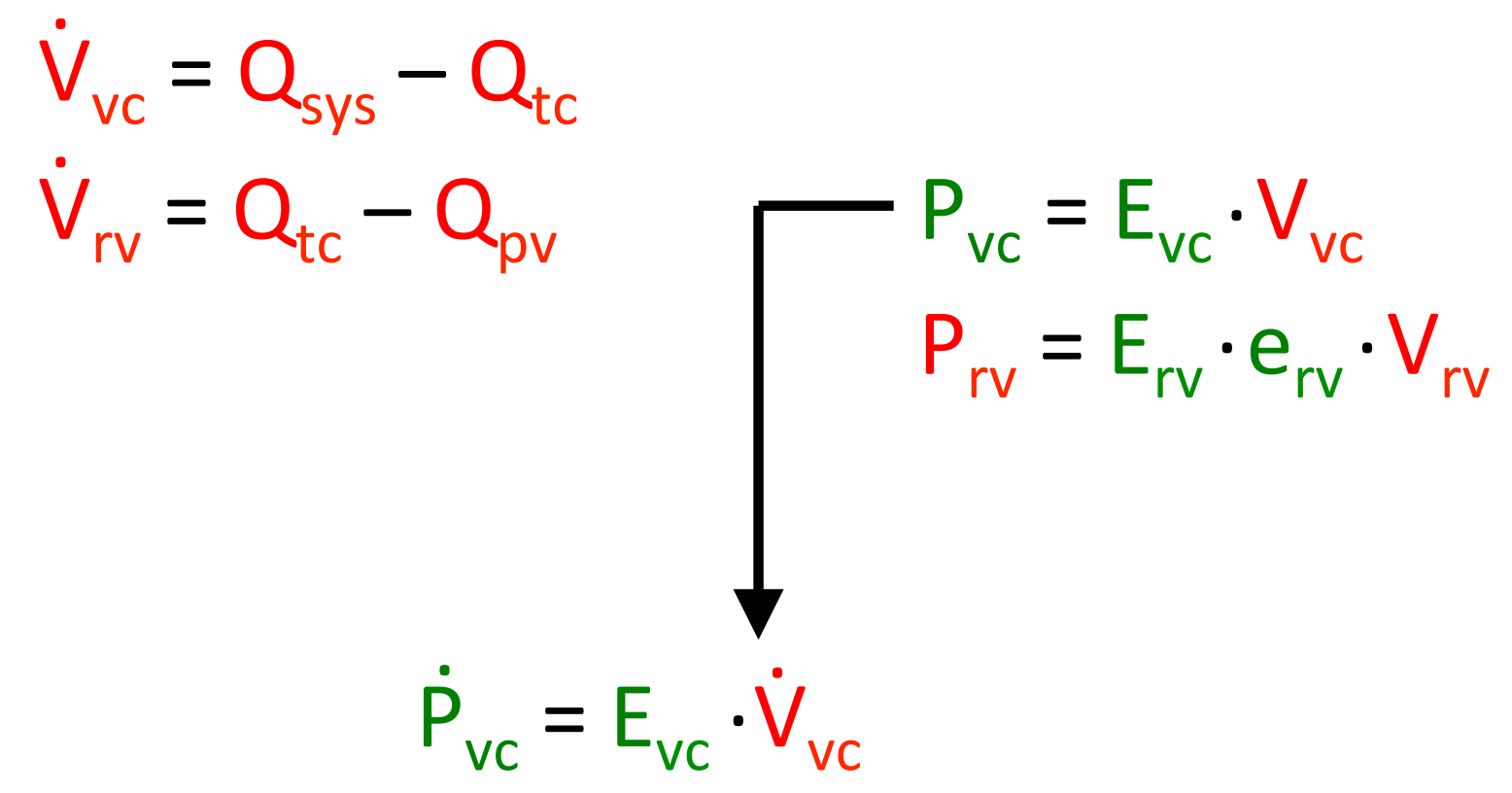

$$
\begin{aligned}
& Q_{s y s}=\frac{P_{a o}-P_{v c}}{R_{s y s}} \\
& Q_{t c}=\frac{P_{v c}-P_{r v}}{R_{t c}} \\
& Q_{p v}=0
\end{aligned}
$$




\section{During cardiac filling: $P_{v c}>P_{r v}$ and $P_{r v}<P_{p a}$}

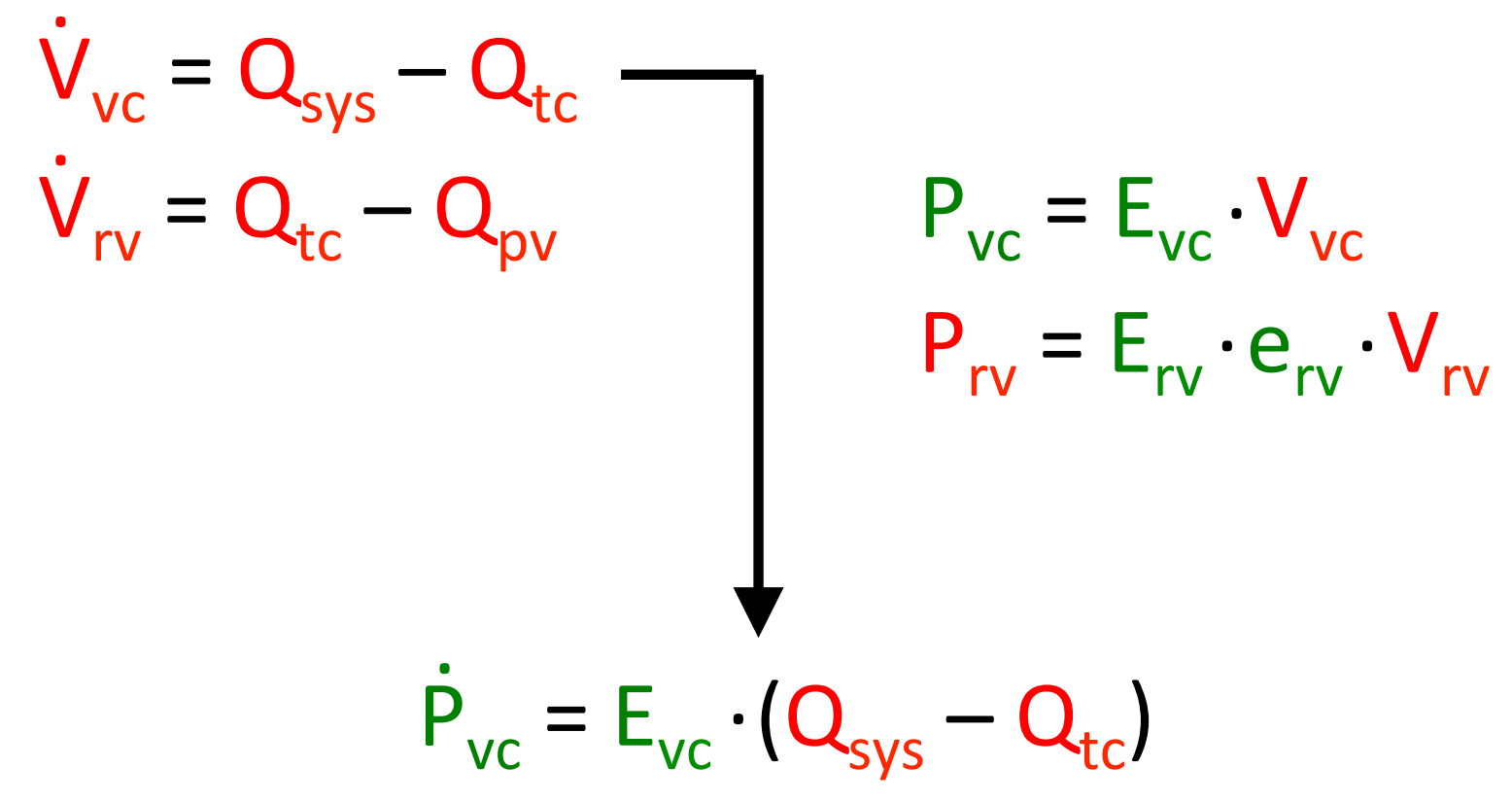

$$
\begin{aligned}
& Q_{s y s}=\frac{P_{a o}-P_{v c}}{R_{s y s}} \\
& Q_{t c}=\frac{P_{v c}-P_{r v}}{R_{t c}} \\
& Q_{p v}=0
\end{aligned}
$$




\section{During cardiac filling: $P_{v c}>P_{r v}$ and $P_{r v}<P_{p a}$}

$$
\begin{aligned}
& \dot{\mathrm{V}}_{\mathrm{vc}}=\mathrm{Q}_{\mathrm{sys}}-\mathrm{Q}_{\mathrm{tc}} \\
& \dot{\mathrm{V}}_{\mathrm{rv}}=\mathrm{Q}_{\mathrm{tc}}-\mathrm{Q}_{\mathrm{pv}} \quad \mathrm{P}_{\mathrm{vc}}=\mathrm{E}_{\mathrm{vc}} \cdot \mathrm{V}_{\mathrm{vc}} \\
& P_{r v}=E_{r v} \cdot e_{r v} \cdot V_{r v} \quad Q_{s y s}=\frac{P_{a o}-P_{v c}}{R_{s y s}} \\
& \dot{P}_{v c}=E_{v c} \cdot\left(\frac{P_{a o}-P_{v c}}{R_{s y s}}-\frac{P_{v c}-P_{r v}}{R_{t c}}\right) \quad Q_{p v}=0
\end{aligned}
$$




\section{During cardiac filling: $P_{v c}>P_{r v}$ and $P_{r v}<P_{p a}$}

$\dot{\mathrm{V}}_{\mathrm{vc}}=\mathrm{Q}_{\mathrm{sys}}-\mathrm{Q}_{\mathrm{tc}}$

$\dot{V}_{\mathrm{rv}}=\mathrm{Q}_{\mathrm{tc}}-\mathrm{Q}_{\mathrm{pv}} \quad \mathrm{P}_{\mathrm{vc}}=\mathrm{E}_{\mathrm{vc}} \cdot \mathrm{V}_{\mathrm{vc}}$

$$
P_{r v}=E_{r v} \cdot e_{r v} \cdot V_{r v}
$$

$Q_{s y s}=\frac{P_{a 0}-P_{v c}}{R_{s y s}}$

$\mathrm{Q}_{\mathrm{tc}}=\frac{\mathrm{P}_{\mathrm{vc}}-\mathrm{P}_{\mathrm{rv}}}{\mathrm{R}_{\mathrm{tc}}}$

$\dot{P}_{v c}=E_{v c} \cdot\left(\frac{P_{a o}-P_{v c}}{R_{s y s}}-\frac{P_{v c}-P_{r v}}{R_{t c}}\right)$

$\mathrm{Q}_{\mathrm{pv}}=0$

$\ddot{P}_{v c}=E_{v c} \cdot\left(\frac{\dot{P}_{\mathrm{ao}}-\dot{\mathrm{P}}_{\mathrm{vc}}}{R_{\mathrm{sys}}}-\frac{\dot{\mathrm{P}}_{\mathrm{vc}}-\dot{\mathrm{P}}_{\mathrm{rv}}}{\mathrm{R}_{\mathrm{tc}}}\right)$ 


\section{During cardiac filling: $P_{v c}>P_{r v}$ and $P_{r v}<P_{p a}$}

$$
\begin{gathered}
\dot{\mathrm{V}}_{\mathrm{vc}}=\mathrm{Q}_{\mathrm{sys}}-\mathrm{Q}_{\mathrm{tc}}=\mathrm{E}_{\mathrm{rv}} \cdot \dot{\mathrm{e}}_{\mathrm{rv}} \cdot \mathrm{V}_{\mathrm{rv}}+\mathrm{E}_{\mathrm{rv}} \cdot \mathrm{e}_{\mathrm{rv}} \cdot \dot{\mathrm{V}}_{\mathrm{rv}} \\
\dot{\mathrm{V}}_{\mathrm{rv}}=\mathrm{Q}_{\mathrm{tc}}-\mathrm{Q}_{\mathrm{pv}} \\
\begin{array}{l}
\mathrm{P}_{\mathrm{vc}}=\mathrm{E}_{\mathrm{vc}} \cdot \mathrm{V}_{\mathrm{vc}} \\
\mathrm{P}_{\mathrm{rv}}=\mathrm{E}_{\mathrm{rv}} \cdot \mathrm{e}_{\mathrm{rv}} \cdot \mathrm{V}_{\mathrm{rv}}
\end{array} \\
\mathrm{Q}_{\mathrm{sys}}=\frac{\mathrm{P}_{\mathrm{ao}}-\mathrm{P}_{\mathrm{vc}}}{\mathrm{R}_{\mathrm{sys}}} \\
\dot{\mathrm{P}}_{\mathrm{vc}}=\mathrm{E}_{\mathrm{vc}} \cdot\left(\frac{\mathrm{P}_{\mathrm{ao}}-\mathrm{P}_{\mathrm{vc}}}{\mathrm{R}_{\mathrm{sys}}}-\frac{\mathrm{P}_{\mathrm{vc}}-\mathrm{P}_{\mathrm{rv}}}{\mathrm{R}_{\mathrm{rc}}}\right. \\
\ddot{\mathrm{P}}_{\mathrm{vc}}=\mathrm{E}_{\mathrm{vc}} \cdot\left(\frac{\dot{\mathrm{P}}_{\mathrm{ao}}-\dot{\mathrm{P}}_{\mathrm{vc}}}{\mathrm{R}_{\mathrm{sys}}}-\frac{\dot{\mathrm{P}}_{\mathrm{vc}}-\dot{\mathrm{P}}_{\mathrm{rv}}}{\mathrm{R}_{\mathrm{tc}}}\right)
\end{gathered}
$$




\section{During cardiac filling: $P_{v c}>P_{r v}$ and $P_{r v}<P_{p a}$}

$$
\begin{aligned}
& \dot{\mathrm{P}}_{\mathrm{rv}}=\mathrm{E}_{\mathrm{rv}} \cdot \dot{\mathrm{e}}_{\mathrm{rv}} \cdot \mathrm{V}_{\mathrm{rv}}+\mathrm{E}_{\mathrm{rv}} \cdot \mathrm{e}_{\mathrm{rv}} \cdot \dot{\mathrm{V}}_{\mathrm{rv}} \\
& \dot{\mathrm{V}}_{\mathrm{vc}}=\mathrm{Q}_{\mathrm{sys}}-\mathrm{Q}_{\mathrm{tc}} \\
& \dot{\mathrm{V}}_{\mathrm{rv}}=\frac{\mathrm{P}_{\mathrm{vc}}-\mathrm{P}_{\mathrm{rv}}}{\mathrm{R}_{\mathrm{tc}}} \\
& 41 \quad P_{r v}=E_{r v} \cdot e_{r v} \cdot V_{r v} \\
& P_{v c}=E_{v c} \cdot V_{v c} \\
& Q_{\text {sys }}=\frac{P_{a o}-P_{v c}}{R_{\text {sys }}} \\
& \mathrm{Q}_{\mathrm{tc}}=\frac{\mathrm{P}_{\mathrm{vc}}-\mathrm{P}_{\mathrm{rv}}}{\mathrm{R}_{\mathrm{tc}}} \\
& \dot{P}_{v c}=E_{v c} \cdot\left(\frac{P_{a o}-P_{v c}}{R_{s y s}}-\frac{P_{v c}-P_{r v}}{R_{t c}}\right) \searrow Q_{p v}=0 \\
& \ddot{\mathrm{P}}_{\mathrm{vc}}=\mathrm{E}_{\mathrm{vc}} \cdot\left(\frac{\dot{\mathrm{P}}_{\mathrm{ao}}-\dot{\mathrm{P}}_{\mathrm{vc}}}{\mathrm{R}_{\mathrm{sys}}}-\frac{\dot{\mathrm{P}}_{\mathrm{vc}}-\dot{\mathrm{P}}_{\mathrm{rv}}}{\mathrm{R}_{\mathrm{tc}}}\right)
\end{aligned}
$$




\section{During cardiac filling: $P_{v c}>P_{r v}$ and $P_{r v}<P_{p a}$}

$$
\begin{gathered}
\dot{P}_{r v}=E_{r v} \cdot \dot{e}_{r v} \cdot V_{r v}+E_{r v} \cdot e_{r v} \cdot \dot{V}_{r v} \\
\dot{V}_{r v}=\frac{P_{v c}-P_{r v}}{R_{t c}} \quad P_{r v}=E_{r v} \cdot e_{r v} \cdot V_{r v}
\end{gathered}
$$

$$
\begin{aligned}
& \dot{P}_{v c}=E_{v c} \cdot\left(\frac{P_{a o}-P_{v c}}{R_{s y s}}-\frac{P_{v c}-P_{r v}}{R_{t c}}\right) \\
& \ddot{P}_{v c}=E_{v c} \cdot\left(\frac{\dot{P}_{a o}-\dot{P}_{v c}}{R_{s y s}}-\frac{\dot{P}_{v c}-\dot{P}_{r v}}{R_{t c}}\right)
\end{aligned}
$$




\section{During cardiac filling: $P_{v c}>P_{r v}$ and $P_{r v}<P_{p a}$}

$$
\begin{aligned}
& R_{t c}=\frac{\dot{P}_{v c} \cdot e_{r v} \cdot E_{v c} \cdot R_{s y s}-\dot{e}_{r v} \cdot E_{v c} \cdot P_{v c} \cdot R_{s y s}+e_{r v}^{2} \cdot E_{r v} \cdot\left(E_{v c} \cdot\left(P_{v c}-P_{a o}\right)+\right.}{\dot{P}_{a o} \cdot e_{r v} \cdot E_{v c}+\dot{e}_{r v} \cdot E_{v c} \cdot\left(P_{v c}-P_{a o}\right)-\ddot{P}_{v c} \cdot e_{r v} \cdot R_{s y s}+\dot{P}_{v c} \cdot\left(\dot{e}_{r v} \cdot R_{s y}\right.} \\
& P_{r v}=\frac{e_{r v} \cdot\left(e_{r v} \cdot E_{r v} \cdot\left(E_{v c} \cdot\left(P_{v c}-P_{a o}\right)+\dot{P}_{v c} \cdot R_{s y s}\right)^{2}+E_{v c} \cdot R_{s y s} \cdot\left(P_{a o} \cdot E_{v c} \cdot\right.\right.}{E_{v c} \cdot R_{s y s} \cdot\left(\dot{P}_{a o} \cdot e_{r v} \cdot E_{v c}+\dot{e}_{r v} \cdot E_{v c} \cdot\left(P_{v c}-P_{a o}\right)-\ddot{P}_{v c} \cdot e_{r v} \cdot R_{s y s}+P_{v}\right.} \\
& V_{r v}=\frac{e_{r v} \cdot E_{r v} \cdot\left(E_{v c} \cdot\left(P_{v c}-P_{a o}\right)+\dot{P}_{v c} \cdot R_{s y s}\right)^{2}+E_{v c} \cdot R_{s y s} \cdot\left(\dot{P}_{a o} \cdot E_{v c} \cdot P_{v c}\right.}{E_{r v} \cdot E_{v c} \cdot R_{s y s} \cdot\left(\dot{P}_{a o} \cdot e_{r v} \cdot E_{v c}+\dot{e}_{r v} \cdot E_{v c} \cdot\left(P_{v c}-P_{a o}\right)-\ddot{P}_{v c} \cdot e_{r v} \cdot R_{s y s}\right.} \\
& \dot{P}_{r v}=\frac{\left(\dot{P}_{a o}-\dot{P}_{v c}\right) \cdot e_{r v}^{2} \cdot E_{r v} \cdot E_{v c}^{2} \cdot\left(P_{a o}-P_{v c}\right)+E_{v c} \cdot\left(\dot{P}_{v c}^{2} \cdot e_{r v}^{2} \cdot E_{r v}-\dot{e}_{r v} \cdot \dot{P}_{v c} \cdot R_{s y s} \cdot\left(\dot{P}_{a o} \cdot e_{r v} \cdot E_{v c}+\dot{e}_{r v} \cdot E_{v c} \cdot\left(P_{v c}-P_{a o}\right)-\ddot{P}_{v c} \cdot e_{r v} \cdot R_{s y s}+\dot{P}_{v}\right.\right.}{E_{v}} \\
& \dot{\mathrm{V}}_{\mathrm{rv}}=\frac{\mathrm{P}_{\mathrm{ao}}-\mathrm{P}_{\mathrm{vc}}}{\mathrm{R}_{\mathrm{sys}}}-\frac{\dot{\mathrm{P}}_{\mathrm{vc}}}{\mathrm{E}_{\mathrm{vc}}}
\end{aligned}
$$




\section{During cardiac filling: $P_{v c}>P_{r v}$ and $P_{r v}<P_{p a}$}

$\dot{\mathrm{V}}_{\mathrm{lv}}=\mathrm{Q}_{\mathrm{mt}}-\mathrm{Q}_{\mathrm{av}}$

$\dot{\mathrm{V}}_{\mathrm{ao}}=\mathrm{Q}_{\mathrm{av}}-\mathrm{Q}_{\mathrm{sys}}$

$\dot{\mathrm{V}}_{\mathrm{vc}}=\mathrm{Q}_{\mathrm{sys}}-\mathrm{Q}_{\mathrm{tc}}$

$\dot{\mathrm{V}}_{\mathrm{rv}}=\mathrm{Q}_{\mathrm{tc}}-\mathrm{Q}_{\mathrm{pv}}$

$\dot{\mathrm{V}}_{\mathrm{pa}}=\mathrm{Q}_{\mathrm{pv}}-\mathrm{Q}_{\mathrm{pul}}$

$\dot{\mathrm{V}}_{\mathrm{pu}}=\mathrm{Q}_{\mathrm{pul}}-\mathrm{Q}_{\mathrm{mt}}$

$\mathrm{SBV}=\mathrm{V}_{\mathrm{lv}}+\mathrm{V}_{\mathrm{ao}}+\mathrm{V}_{\mathrm{vc}}+\mathrm{V}_{\mathrm{rV}}+\mathrm{V}_{\mathrm{pa}}+\mathrm{V}_{\mathrm{pu}}$

$S V=\int_{0}^{T} Q d t$

$$
\begin{aligned}
& Q_{m t}=\frac{r\left(P_{p u}-P_{l v}\right)}{R_{m t}} \\
& Q_{a v}=\frac{r\left(P_{l v}-P_{a o}\right)}{R_{a v}} \\
& Q_{s y s}=\frac{P_{a o}-P_{v c}}{R_{s y s}} \\
& Q_{t c}=\frac{r\left(P_{v c}-P_{r v v}\right)}{R_{t c}} \\
& Q_{p v}=\frac{r\left(P_{r v}-P_{p a}\right)}{R_{p v}} \\
& Q_{p u l}=\frac{P_{p a}-P_{p u}}{R_{p u l}}
\end{aligned}
$$




\section{During cardiac filling: $P_{v c}>P_{r v}$ and $P_{r v}<P_{p a}$}

$\dot{\mathrm{V}}_{\mathrm{lv}}=\mathrm{Q}_{\mathrm{mt}}-\mathrm{Q}_{\mathrm{av}}$

$\dot{\mathrm{V}}_{\mathrm{ao}}=\mathrm{Q}_{\mathrm{av}}-\mathrm{Q}_{\mathrm{sys}}$

$\dot{\mathrm{V}}_{\mathrm{vc}}=\mathrm{Q}_{\mathrm{sys}}-\mathrm{Q}_{\mathrm{tc}}$

$\dot{\mathrm{V}}_{\mathrm{rv}}=\mathrm{Q}_{\mathrm{tc}}-\mathrm{Q}_{\mathrm{pv}}$

$\dot{\mathrm{V}}_{\mathrm{pa}}=\mathrm{Q}_{\mathrm{pv}}-\mathrm{Q}_{\mathrm{pul}}$

$\dot{\mathrm{V}}_{\mathrm{pu}}=\mathrm{Q}_{\mathrm{pul}}-\mathrm{Q}_{\mathrm{mt}}$

$\mathrm{SBV}=\mathrm{V}_{\mathrm{lv}}+\mathrm{V}_{\mathrm{ao}}+\mathrm{V}_{\mathrm{vc}}+\mathrm{V}_{\mathrm{rV}}+\mathrm{V}_{\mathrm{pa}}+\mathrm{V}_{\mathrm{pu}}$

$S V=\int_{0}^{T} Q d t$

$$
\begin{aligned}
& Q_{m t}=\frac{r\left(P_{p u}-P_{l v}\right)}{R_{m t}} \\
& Q_{a v}=\frac{r\left(P_{l v}-P_{a o}\right)}{R_{a v}} \\
& Q_{s y s}=\frac{P_{a o}-P_{v c}}{R_{s y s}} \\
& Q_{t c}=\frac{r\left(P_{v c}-P_{r v}\right)}{R_{t c}} \\
& Q_{p v}=\frac{r\left(P_{r v}-P_{p a}\right)}{R_{p v}} \\
& Q_{p u l}=\frac{P_{p a}-P_{p u}}{R_{p u l}}
\end{aligned}
$$




\section{During cardiac filling: $\mathrm{P}_{\mathrm{pu}}>\mathrm{P}_{\mathrm{lv}}$ and $\mathrm{P}_{\mathrm{lv}}<\mathrm{P}_{\mathrm{ao}}$}

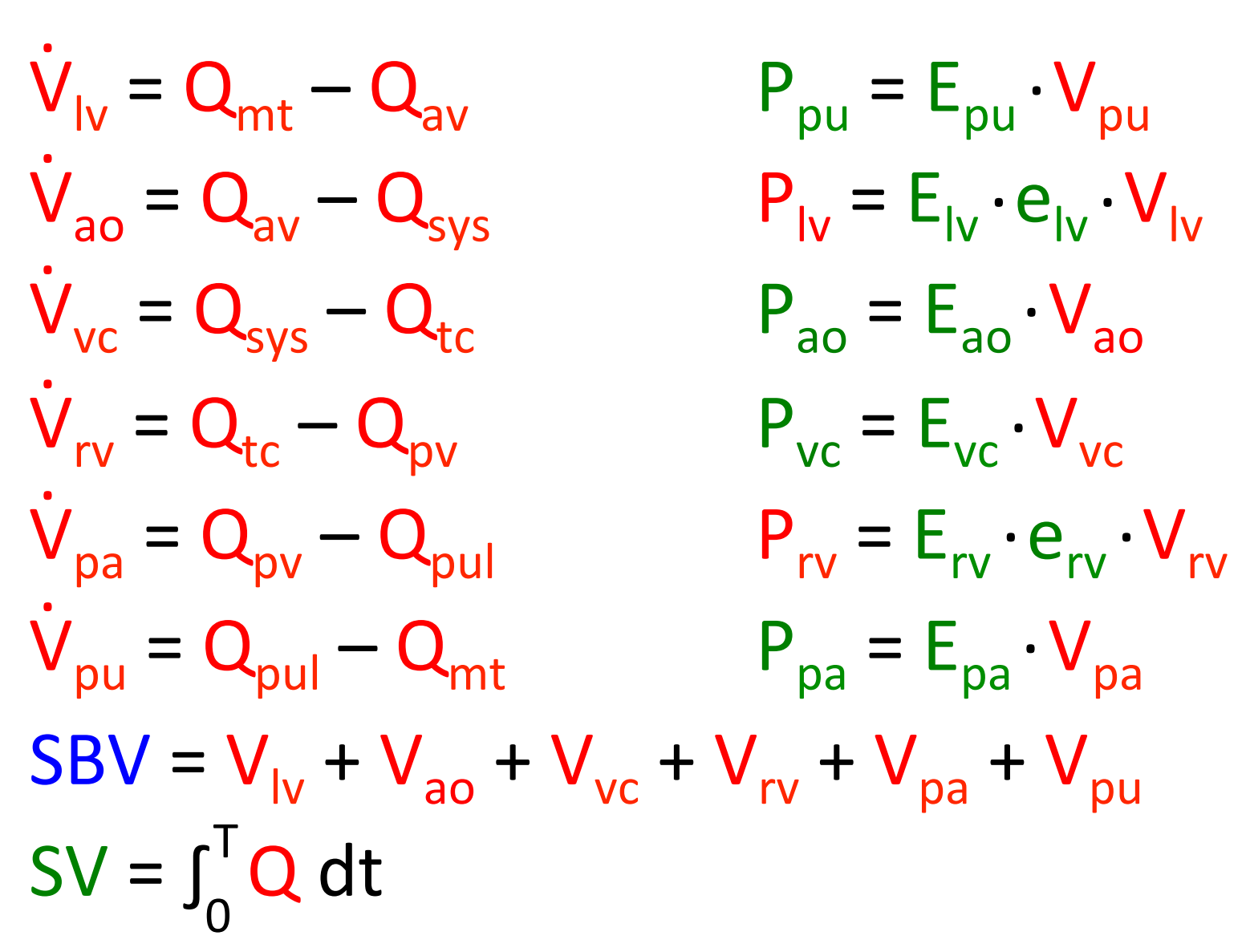

$$
\begin{aligned}
& Q_{m t}=\frac{r\left(P_{p u}-P_{l v}\right)}{R_{m t}} \\
& Q_{a v}=\frac{r\left(P_{l v}-P_{a o}\right)}{R_{a v}} \\
& Q_{s y s}=\frac{P_{a o}-P_{v c}}{R_{s y s}} \\
& Q_{t c}=\frac{r\left(P_{v c}-P_{r v}\right)}{R_{t c}} \\
& Q_{p v}=\frac{r\left(P_{r v}-P_{p a}\right)}{R_{p v}} \\
& Q_{p u l}=\frac{P_{p a}-P_{p u}}{R_{p u l}}
\end{aligned}
$$


During cardiac ejection: $\mathrm{P}_{\mathrm{pu}}<\mathrm{P}_{\mathrm{lv}}$ and $\mathrm{P}_{\mathrm{lv}}>\mathrm{P}_{\mathrm{ao}}$

$\dot{\mathrm{V}}_{\mathrm{lv}}=\mathrm{Q}_{\mathrm{mt}}-\mathrm{Q}_{\mathrm{av}}$

$\dot{\mathrm{V}}_{\mathrm{ao}}=\mathrm{Q}_{\mathrm{av}}-\mathrm{Q}_{\mathrm{sys}}$

$\dot{\mathrm{V}}_{\mathrm{vc}}=\mathrm{Q}_{\mathrm{sys}}-\mathrm{Q}_{\mathrm{tc}}$

$\dot{\mathrm{V}}_{\mathrm{rv}}=\mathrm{Q}_{\mathrm{tc}}-\mathrm{Q}_{\mathrm{pv}}$

$\dot{\mathrm{V}}_{\mathrm{pa}}=\mathrm{Q}_{\mathrm{pv}}-\mathrm{Q}_{\mathrm{pul}}$

$\dot{\mathrm{V}}_{\mathrm{pu}}=\mathrm{Q}_{\mathrm{pul}}-\mathrm{Q}_{\mathrm{mt}}$

$\mathrm{SBV}=\mathrm{V}_{\mathrm{lv}}+\mathrm{V}_{\mathrm{ao}}+\mathrm{V}_{\mathrm{vc}}+\mathrm{V}_{\mathrm{rV}}+\mathrm{V}_{\mathrm{pa}}+\mathrm{V}_{\mathrm{pu}}$

$S V=\int_{0}^{T} Q d t$

$$
\begin{aligned}
& Q_{m t}=\frac{r\left(P_{p u}-P_{l v}\right)}{R_{m t}} \\
& Q_{a v}=\frac{r\left(P_{l v}-P_{a o}\right)}{R_{a v}} \\
& Q_{s y s}=\frac{P_{a o}-P_{v c}}{R_{s y s}} \\
& Q_{t c}=\frac{r\left(P_{v c}-P_{r v}\right)}{R_{t c}} \\
& Q_{p v}=\frac{r\left(P_{r v}-P_{p a}\right)}{R_{p v}} \\
& Q_{p u l}=\frac{P_{p a}-P_{p u}}{R_{p u l}}
\end{aligned}
$$


During cardiac ejection: $P_{v c}<P_{r v}$ and $P_{r v}>P_{r}$

pa

$\dot{\mathrm{V}}_{\mathrm{lv}}=\mathrm{Q}_{\mathrm{mt}}-\mathrm{Q}_{\mathrm{av}}$

$\dot{\mathrm{V}}_{\mathrm{ao}}=\mathrm{Q}_{\mathrm{av}}-\mathrm{Q}_{\text {sys }}$

$\dot{\mathrm{V}}_{\mathrm{vc}}=\mathrm{Q}_{\mathrm{sys}}-\mathrm{Q}_{\mathrm{tc}}$

$\dot{\mathrm{V}}_{\mathrm{rv}}=\mathrm{Q}_{\mathrm{tc}}-\mathrm{Q}_{\mathrm{pv}}$

$\dot{\mathrm{V}}_{\mathrm{pa}}=\mathrm{Q}_{\mathrm{pv}}-\mathrm{Q}_{\mathrm{pul}}$

$\dot{\mathrm{V}}_{\mathrm{pu}}=\mathrm{Q}_{\mathrm{pul}}-\mathrm{Q}_{\mathrm{mt}}$

$\mathrm{SBV}=\mathrm{V}_{\mathrm{lv}}+\mathrm{V}_{\mathrm{ao}}+\mathrm{V}_{\mathrm{vc}}+\mathrm{V}_{\mathrm{rV}}+\mathrm{V}_{\mathrm{pa}}+\mathrm{V}_{\mathrm{pu}}$

$S V=\int_{0}^{T} Q d t$

$$
\begin{aligned}
& Q_{m t}=\frac{r\left(P_{p u}-P_{l v}\right)}{R_{m t}} \\
& Q_{a v}=\frac{r\left(P_{l v}-P_{a o}\right)}{R_{a v}} \\
& Q_{s y s}=\frac{P_{a o}-P_{v c}}{R_{s y s}} \\
& Q_{t c}=\frac{r\left(P_{v c}-P_{r v}\right)}{R_{t c}} \\
& Q_{p v}=\frac{r\left(P_{r v}-P_{p a}\right)}{R_{p v}} \\
& Q_{p u l}=\frac{P_{p a}-P_{p u}}{R_{p u l}}
\end{aligned}
$$




\section{During cardiac filling}

$$
\begin{aligned}
& P_{p u}=E_{p u} \cdot V_{p u} \\
& P_{a o}=E_{a o} \cdot V_{a o} \\
& P_{v c}=E_{v c} \cdot V_{v c}
\end{aligned}
$$$$
\mathrm{P}_{\mathrm{pa}}=\mathrm{E}_{\mathrm{pa}} \cdot \mathrm{V}_{\mathrm{pa}}
$$

$\mathrm{SBV}=\mathrm{V}_{\mathrm{lv}}+\mathrm{V}_{\mathrm{ao}}+\mathrm{V}_{\mathrm{vc}}+\mathrm{V}_{\mathrm{rV}}+\mathrm{V}_{\mathrm{pa}}+\mathrm{V}_{\mathrm{pu}}$ 


\section{During cardiac filling}

$$
\begin{aligned}
& P_{p u}=E_{p u} \cdot V_{p u} \\
& P_{a o}=E_{a o} \cdot V_{a o} \\
& P_{v c}=E_{v c} \cdot V_{v c}
\end{aligned}
$$$$
P_{p a}=E_{p a} \cdot V_{p a}
$$

$S B V=V_{l v}+V_{a o}+V_{v c}+V_{r v}+V_{p a}+V_{p u}$ 


\section{During cardiac filling}

$$
\begin{aligned}
& P_{p u}=E_{p u} \cdot V_{p u} \\
& P_{a o}=E_{a o} \cdot V_{a o} \\
& P_{v c}=E_{v c} \cdot V_{v c}
\end{aligned}
$$

$$
P_{p a}=E_{p a} \cdot V_{p a}
$$

$S B V=V_{l v}+V_{a o}+V_{v c}+V_{r V}+V_{p a}+V_{p u}$ 


\section{During cardiac filling}

$$
\begin{aligned}
& P_{p u}=E_{p u} \cdot V_{p u} \\
& P_{a o}=E_{a o} \cdot V_{a o} \\
& P_{v c}=E_{v c} \cdot V_{v c}
\end{aligned}
$$

$$
P_{p a}=E_{p a} \cdot V_{p a}
$$

$S B V=V_{l v}+V_{a o}+V_{v c}+V_{r v}+V_{p a}+V_{p u}$ 


\section{During cardiac filling}

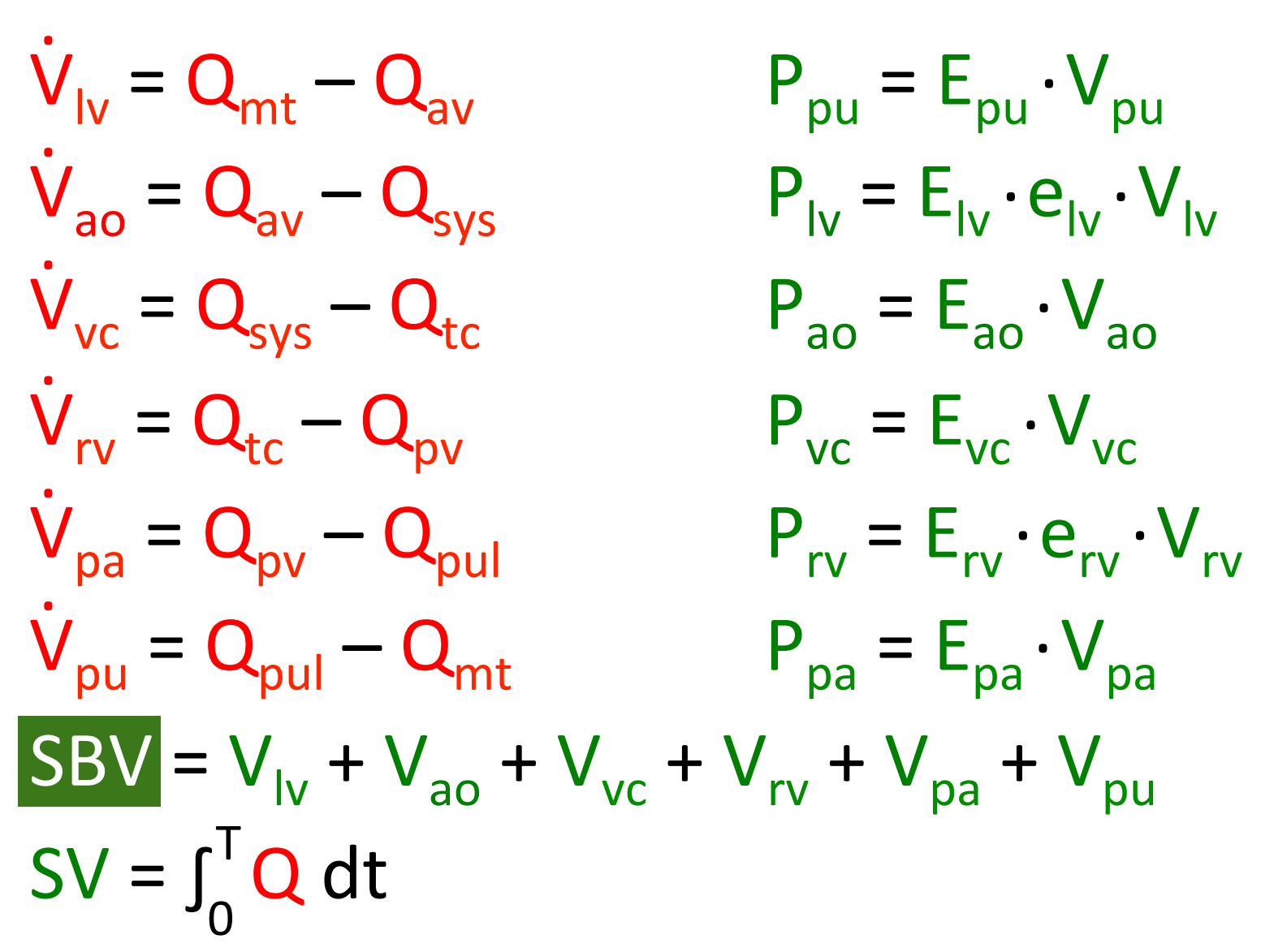

$$
\begin{aligned}
& Q_{m t}=\frac{r\left(P_{p u}-P_{l v}\right)}{R_{m t}} \\
& Q_{a v}=\frac{r\left(P_{l v}-P_{a o}\right)}{R_{a v}} \\
& Q_{s y s}=\frac{P_{a o}-P_{v c}}{R_{s y s}} \\
& Q_{t c}=\frac{r\left(P_{v c}-P_{r v}\right)}{R_{t c}} \\
& Q_{p v}=\frac{r\left(P_{r v}-P_{p a}\right)}{R_{p v}} \\
& Q_{p u l}=\frac{P_{p a}-P_{p u}}{R_{p u l}}
\end{aligned}
$$




\section{Results}

The 13 model parameters can be uniquely retrieved from (perfect) measurements of

- aortic pressure,

- pulmonary artery pressure,

- vena cava pressure,

- pulmonary vein pressure,

- stroke volume and knowledge of the two driver functions. 


\section{Practical considerations}

The 13 model parameters can be uniquely retrieved from (perfect) measurements of

- aortic pressure,

- pulmonary artery pressure,

- vena cava pressure,

- pulmonary vein pressure,

- stroke volume

and knowledge of the two driver functions. 


\section{Practical considerations}

The 13 model parameters can be uniquely retrieved from (perfect) measurements of

- aortic pressure,

- pulmonary artery pressure,

- vena cava pressure,

- pulmonary vein pressure,

- stroke volume

and knowledge of the two driver functions. 


\section{Practical considerations}

The 13 model parameters can be uniquely retrieved from (perfect) measurements of

- aortic pressure,

- pulmonary artery pressure,

- vena cava pressure,

- pulmonary vein pressure,

- stroke volume

and knowledge of the two driver functions. 


\section{Practical considerations}

The 13 model parameters can be uniquely retrieved from (perfect) measurements of

- aortic pressure,

- pulmonary artery pressure,

- vena cava pressure,

- pulmonary vein pressure,

- stroke volume

and knowledge of the two driver functions. 


\section{Practical considerations}

The 13 model parameters can be uniquely retrieved from (perfect) measurements of

- aortic pressure,

- pulmonary artery pressure,

- vena cava pressure,

- pulmonary vein pressure,

- stroke volume

and knowledge of the two driver functions. 


\section{Practical considerations}

The 13 model parameters can be uniquely retrieved from (perfect) measurements of

- aortic pressure,

- pulmonary artery pressure,

- vena cava pressure,

- pulmonary vein pressure,

- stroke volume and knowledge of the two driver functions. 


\section{Conclusions}

- The six-chamber CVS model is structurally globally identifiable from an output set containing only a limited number of clinically available measurements. 


\section{Conclusions}

- The six-chamber CVS model is structurally globally identifiable from an output set containing only a limited number of clinically available measurements.

- The model parameters computed from this dataset are theoretically unique. 


\section{Conclusions}

- The six-chamber CVS model is structurally globally identifiable from an output set containing only a limited number of clinically available measurements.

- The model parameters computed from this dataset are theoretically unique.

- Hence, the parameter values are fully suitable to be used for diagnosis. 
Thanks for your attention! 